\title{
An Optimized Sparse Approximate Matrix Multiply for Matrices with Decay
}

\author{
Nicolas Bock ${ }^{*}$ and Matt Challacombe $\left.\right|^{\dagger}$ \\ Group T-1, Theoretical Division, Los Alamos National Laboratory, Los Alamos, NM 87544
}

(Dated: September 5, 2012)

\begin{abstract}
We present an optimized single-precision implementation of the Sparse Approximate Matrix Multiply (SpAMM) [M. Challacombe and N. Bock, arXiv 1011.3534 (2010)], a fast algorithm for matrixmatrix multiplication for matrices with decay that achieves an $\mathcal{O}(n \log n)$ computational complexity with respect to matrix dimension $n$. We find that the max norm of the error achieved with a SpAMM tolerance below $2 \times 10^{-8}$ is lower than that of the single-precision SGEMM for dense quantum chemical matrices, while outperforming SGEMM with a cross-over already for small matrices $(n \sim 1000)$. Relative to naive implementations of SpAMM using Intel's Math Kernel Library (MKL) or AMD's Core Math Library (ACML), our optimized version is found to be significantly faster. Detailed performance comparisons are made for quantum chemical matrices with differently structured sub-blocks. Finally, we discuss the potential of improved hardware prefetch to yield 2-3x speedups.
\end{abstract}

Keywords: Sparse Approximate Matrix Multiply; Sparse Linear Algebra; SpAMM; Reduced Complexity Algorithm; Quantum Chemistry; $N$-Body; Matrices with Decay

\section{INTRODUCTION}

For large dense linear algebra problems, the computational advantage offered by fast matrix-matrix multiplication can be substantial, even with seemingly small gains in asymptotic complexity ${ }^{1}$. Relative to conventional multiplication which is $\mathcal{O}\left(n^{3}\right)$, Strassen's algorithm 130 achieves $\mathcal{O}\left(n^{2.8}\right)$, while the Coppersmith and Winograd method 48 and the method of Williams' [136] are bound by $\mathcal{O}\left(n^{2.3755}\right)$ and $\mathcal{O}\left(n^{2.3727}\right)$, respectively. For these dense methods, balancing the trade-off between cost, complexity and error is an active area of research [51, 52, 154. On the other hand, large sparse problems are typically handled with conventional sparse matrix techniques, with only small concessions between multiplication algorithms.

Intermediate to these regimes, a wide class of problems exist that involve matrices with decay, occurring in the construction of matrix functions [18, notably the matrix inverse 20, 50, the matrix exponential 88, and in the case of electronic structure theory, the Heaviside step function (spectral projector) [17, 19, 34, 35, 107, 118. A matrix $A$ is said to decay when its matrix elements decrease exponentially, as $\left|a_{i, j}\right|<c \lambda^{|i-j|}$, or algebraically as $\left|a_{i, j}\right|<c /\left(|i-j|^{\lambda}+1\right)$ with separation $|i-j|$. In nonsynthetic cases, the separation $|i-j|$ may correspond to an underlying physical distance $\left|\vec{r}_{i}-\vec{r}_{j}\right|$, e.g. of basis functions, finite elements, etc. 17. Note, matrices with decay are typically not sparse, but exhibit a structure

\footnotetext{
*Electronic address: nbock@lanl.gov

$\dagger$ Electronic address: mchalla@lanl.gov

1 Throughout, we refer to the asymptotic bounds of an algorithm in big- $\mathcal{O}$ notation when using the term "complexity", while the term "performance" is reserved for the actual runtime or cycle count of an implementation. Since performance is affected by the complexity of the underlying algorithm and computer and implementation specific details, complexity and performance are not necessarily the same.
}
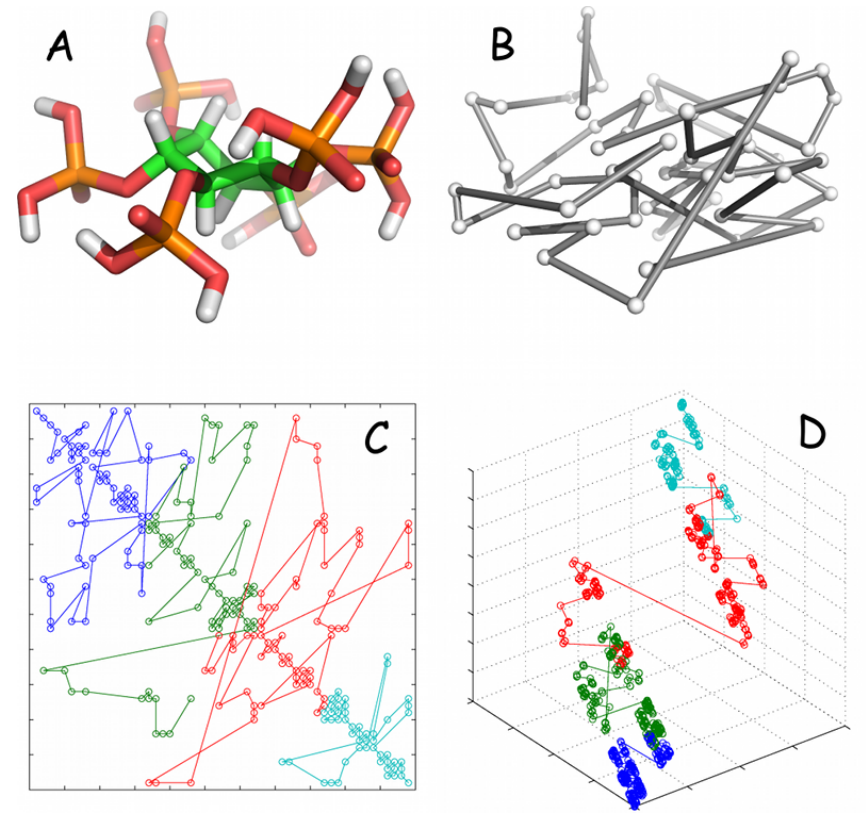

FIG. 1: Space filling curves (SFC) map atoms in Cartesian space (A) onto an index that is locality preserving (B), leading to clustering of matrix elements due to decay and the underlying physical system, shown for the sparsified case (C). In addition the locality preserving mechanisms of the SFC can be extended to 2-D $(i j)$ data decomposition of matrices, $(\mathrm{C})$, as well as three-dimensional decomposition $(i j k)$ in product space of the matrix-matrix multiply (D).

that can be exploited to yield an approximate sparse algorithm that exhibits a reduced complexity.

Reordering the physical indices $\vec{r}_{i}$ along a locality preserving space-filling curve (SFC), such as the Hilbert 84 or the Peano curve [121, maps elements that are close in three dimensions onto an ordered list [123, 139, 143, leading to effective clustering of submatrices by order of magnitude [35]. Figure 1 illustrates this point for quantum chemistry applications where the atom indices of the 


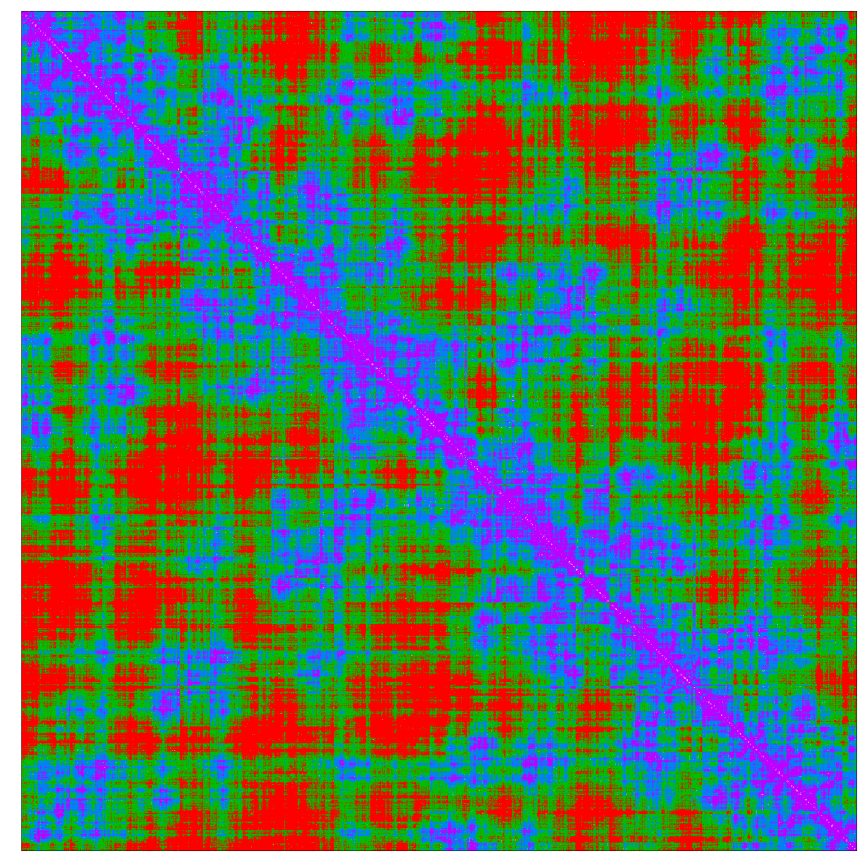

FIG. 2: The decay of matrix element magnitudes of a converged spectral projector (density matrix) for a $\left(\mathrm{H}_{2} \mathrm{O}\right)_{300}$ water cluster at the RHF/6-31G ${ }^{* *}$ level of theory $(n=7500)$, where the molecular geometry has been reordered with a space filling Hilbert curve. The different colors indicate different matrix element magnitudes; red: $\left[0,10^{-8}\right)$; green: $\left[10^{-8}, 10^{-6}\right)$; blue: $\left[10^{-6}, 10^{-2}\right)$; violet: $\left[10^{-2}, 1\right]$, corresponding to approximate exponential decay.

molecule shown in panel A are ordered along the SFC, shown in panel B, leading to the clustered structure of matrix element magnitudes shown schematically in panel $\mathrm{C}$ of Fig. 1 and specifically in Fig. 2, Extending SFC ordering to the two dimensional vector space of matrix elements $A_{i j}$, shown in panel $\mathrm{C}$, and the three dimensional product space of product contributions $A_{i k} B_{k j}$, shown in panel $\mathrm{D}$, extends the locality preserving properties of SFC to matrix element storage and matrix product execution order. Historically, complexity reduction for matrices with decay has been achieved through "sparsification" or dropping of small matrix elements and the use of conventional sparse matrix kernels [26, 27, 59, 6567, 100. Alternatives such as divide and conquer exist [95, 113, 151, 152, where block diagonal subsystems are "glued" together, but these methods are unable to retain long range coherence effects that can manifest in the spectral projector, show for example in Fig. 2 as crossdiagonal bands. Recently, the authors introduced a fast method for calculating the product of matrices with decay, the Sparse Approximate Matrix Multiply (SpAMM) [37, which is different from truncation in the vector space, $(i j)$ and $(j k)$, in that it hierarchically eliminates insignificant contributions in the product space $(i j k)$, leading to complexity reduction through a sparse product space. The recursive approach is similar to Strassen's algorithm [130] and also to methods of improving locality across memory hierarchies pioneered by Wise and coworkers [6, 56, 105, 145, 148].

SpAMM belongs to a broad class of generalized $N$-Body solvers, "fast" algorithms that achieve reduced complexity through hierarchical approximation, achieving increasingly broad applicability and high performance implementations with surprising commonalities. Related solvers include the database join operation [44, 94, 110, 124, gravitational force summation [140, 141, 143], the Coulomb interaction [40, 126], the fast Gauß transform [14, 78, 129, 137, 149, adaptive mesh refinement [16, 21, 22, the fast multipole method [45, 75, 77] and visualization applications [10, 24, 47, 96, 97, 99, 155.

SpAMM is similar to the $\mathcal{H}$-matrix algebra of Hackbusch and co-workers [72, 81] and the Hierarchically Semiseparable (HSS) representation of Chandrasekaran, $\mathrm{Gu}$ and co-workers [42, 43, where off-diagonal submatrices are treated hierarchically as reduced rank factorizations (truncated SVD), typically structured and grouped to reflect properties of the underlying operators. For problems with rapid decay, truncated SVD may behave in a similar way to simple dropping schemes. However, SpAMM is different than the $\mathcal{H}$-matrix or the HSS algebra as it achieves separation uniquely in the product space and does not rely on a reduced complexity representation of matrices. For very slow decay, where SpAMM is ineffective, the $\mathcal{H}$-matrix or HSS algebra may certainly offer an alternative.

In Ref. [37, a naïve implementation of SpAMM was developed using recursion and a simple unoptimized $4 \times 4 \times 4$ multiplication at the lowest level. In this article we describe an optimized, single-precision, non-recursive kernel of SpAMM that can be introduced at any level of a recursive scheme targeting full precision of the conventional SGEMM kernel. Precision of the SGEMM corresponds roughly to many approximate double-precision $\mathcal{O}(n)$ schemes, which yield $\approx 7$ digits in the total energy and $\approx 5$ digits in the forces, with an additional two-fold savings in time and space. The optimized algorithm includes a hand-coded assembly kernel using single-precision SSE instructions for $4 \times 4 \times 4$ matrix products, demonstrating significantly higher performance than any naïve implementation using vendor tuned BLAS kernels, while yielding superior error control.

Our article is organized as follows: In Sec. II we describe briefly the SpAMM algorithm for the matrix multiply. In Sec. III, we describe in detail our highperformance implementation. In Sec. IV] we describe the benchmark setup and methodologies used. In Sec. V] we present our results for benchmarks on representative density matrices from quantum chemistry calculations performed on two hardware platforms, Intel Xeon X5650 and AMD Opteron 6168. In Sec. VI, we discuss the results and, finally, in Sec.VII we present our conclusions. 


\section{ALGORITHM AND THEORY}

The SGEMM (single precision) function of BLAS calculates the following expression,

$$
C=\alpha A B+\beta C,
$$

where $A, B$, and $C$ are $m \times k, k \times n$, and $m \times n$ matrices, respectively, and $\alpha$ and $\beta$ are scalar parameters. SpAMM calculates the same but is optimized for matrices with decay. In SpAMM we store an $m \times n$ matrix in a quaternary tree (quadtree) data structure [55, an idea developed by Wise et al. [6, 145, 146, Beckman [15, and Samet 122,123 . The quadtree resolves the matrix decay pattern hierarchically and is particularly well suited for the representation of matrices with a clustered structure such as that shown in Fig. 2 and explained in Fig. 1. Furthermore, computational cost for quadtree access exhibits favorable scaling with respect to matrix size [147.

Compared to the more traditional Compressed Sparse Row or Column (CSR or CSC, respectively) data structure, which is typically stored in contiguous arrays, the quadtree can easily be allocated non-contiguously and allows for insertion and deletion without requiring data copy operations. Note that even in the case of more advanced CSR data structures the pointer table is allocated contiguously, see for instance the BCSR data structure of Challacombe [35] which uses blocked data, or the doubly compressed sparse row format (DCSR) of Buluç et al. [29] developed for the efficient representation of hypersparse matrices.

In addition, CSR based implementations face challenges in parallel, currently significantly suppressing performance scaling to large processor counts. In the context of electronic structure calculations for instance, it has been observed that data movement in parallel CSR summation can dominate the parallel construction of sparse Hamiltonians 60. Similarly, the parallel sparse matrixmatrix multiply (SpGEMM) of Buluç et al. [28, 30] becomes communication bound with increasing processor count due to the 2-D decomposition and random permutation of matrix rows and columns that simplify communication and load balancing, but lead to communication cost scaling as in the dense (Cannon's algorithm [33] or SUMMA [134] ) case, $\mathcal{O}(\alpha \sqrt{p}+\beta c n / \sqrt{p})$, where $\alpha$ represents communication latency, $\beta$ communication bandwidth, and $c$ the average number of non-zero elements per row.

There is also a fundamental philosophical difference between SpAMM and CSR based matrix-matrix multiply algorithms with SPGEMM as a state of the art example. On the one hand, SpAMM exploits locality manifest in clustering of matrix elements by order of magnitude (Fig. 2) to achieve a reduced complexity through hierarchical approximation in the product space. On the other hand, the parallel SpGEMM exploits randomization and corresponding loss of locality to achieve load balance. While Buluç's idea has been deployed by a large scale quantum chemistry program [135, the impact of data reordering and loss of locality on scalability would appear to also suffer from communication bottlenecks with same formal complexities as Buluç's. It remains for us to demonstrate that these complexities can be overcome in the SpAMM approach by retaining locality and achieving load balance instead in overdecomposition of recursive task space. In addition, the SpGEMM approach would strongly suppress performance of quantum chemical algorithms due to total loss of (matrix) locality, as well as interoperability problems due to global ordering and reordering of data.

The non-contiguous allocation of a quadtree and the SFC ordering of matrix elements should therefore be more suited for integration with other quantum chemical solvers and in parallel implementations where data and work distribution in a non-shared memory environment is important and data repacking undesirable.

\section{A. Quadtree Data Structure}

Given an $m \times n$ matrix, the depth of the quadtree is given by

$$
d=\left\lceil\max \left(\frac{\log \left(m / n_{b}\right)}{\log 2}, \frac{\log \left(n / n_{b}\right)}{\log 2}\right)\right\rceil,
$$

where $n_{b} \times n_{b}$ is the size of the dense submatrices stored at the bottom of the tree, chosen for performance. To simplify recursion, the matrix is zero padded to square shape of size $n_{p}=n_{b} 2^{d}$. The computational cost of the lookup, insertion, and deletion of matrix elements is $\mathcal{O}\left(\log n_{p}\right)$, independent of the decay pattern. The root tier is denoted as $t=0$ and at each tier $t<d$ of the quadtree, a node links to the subnodes of the $2 \times 2$ submatrix underneath it,

$$
A^{t}=\left(\begin{array}{ll}
A_{11}^{t+1} & A_{12}^{t+1} \\
A_{21}^{t+1} & A_{22}^{t+1}
\end{array}\right)
$$

If a submatrix is zero, its link is set to NULL and the tree terminates at that point. Finally, at the bottom tier $t=d$, each node stores a dense $n_{b} \times n_{b}$ submatrix.

\section{B. SpAMM}

In each node we also store the Frobenius norm of its submatrix, which is given by

$$
\left\|A^{t}\right\|_{F}=\sqrt{\sum_{i, j=1}^{2}\left\|A_{i j}^{t+1}\right\|_{F}^{2} .}
$$

Since the square of the Frobenius norm is additive in its submatrix norms we can construct it recursively starting at the bottom tier. The matrix product is defined 
recursively at each tier as

$$
C_{i j}^{t}=\sum_{k=1}^{2} A_{i k}^{t+1} B_{k j}^{t+1} \quad i, j=1,2,
$$

subject to the SPAMM condition

$$
A_{i k}^{t+1} B_{k j}^{t+1} \equiv 0 \quad \text { if }\left\|A_{i k}^{t+1}\right\|_{F}\left\|B_{k j}^{t+1}\right\|_{F}<\tau,
$$

where $\tau$ is the SpAMM product tolerance.

\section{Matrix Sparsity vs. Product Sparsity}

Matrices with algebraic or exponential decay are not necessarily sparse unless elements are dropped (sparsified). Storage of dense matrices is $\mathcal{O}\left(n^{2}\right)$, while lookup and insertion/deletion is $\mathcal{O}(\log n)$. In practice though, with $\tau>0$, truncation in the product space leads naturally to low level truncation in the vector space of the result, $C$. Likewise, it is possible to apply a very small threshold to the product matrices $A$ and $B$ to maintain an $\mathcal{O}(n \log n)$ computational complexity of the multiply (for further discussion, see Sec. VI). In particular, using a drop tolerance $\epsilon$, with

$$
\epsilon=\frac{\tau}{\max \left(\|A\|_{F},\|B\|_{F}\right)}
$$

is numerically consistent with the SpAMM condition. The accumulation of error due to low level sparsification and product space truncation are certain to be application specific, with a detailed analysis beyond the scope of the current work. Here we consider only errors associated with application of the SpAMM condition to full (dense) matrices.

\section{IMPLEMENTATION}

Figure 3 shows the performance of tuned SGEMM kernels from MKL [3] and ACML [1] with increasing matrix size. For small matrices, the performance is mostly throttled by memory access since memory latency is very large with $\approx 60-100 \mathrm{~ns}$ (at $2.8 \mathrm{GHz}$, this translates into roughly 168-280 cycles) per memory access to main memory 98, Table 2], compared to the latency of an addition or multiplication operation, taking only 3 or 4 cycles [87, Xeon X5650, Appendix C], respectively. In order to mitigate this latency, optimized, cache-aware SGEMM implementations use detailed knowledge of the cache hierarchy and data reordering techniques, such as partitioning and packing into contiguous buffers 68, 70, 80, to replace main memory access with cache access, which is significantly faster (access to L1 data cache takes $\approx 10$ cycles).

\section{A. Cache-Oblivious Algorithms}

A class of alternative approaches is aimed at avoiding knowledge of the cache hierarchy altogether through im-

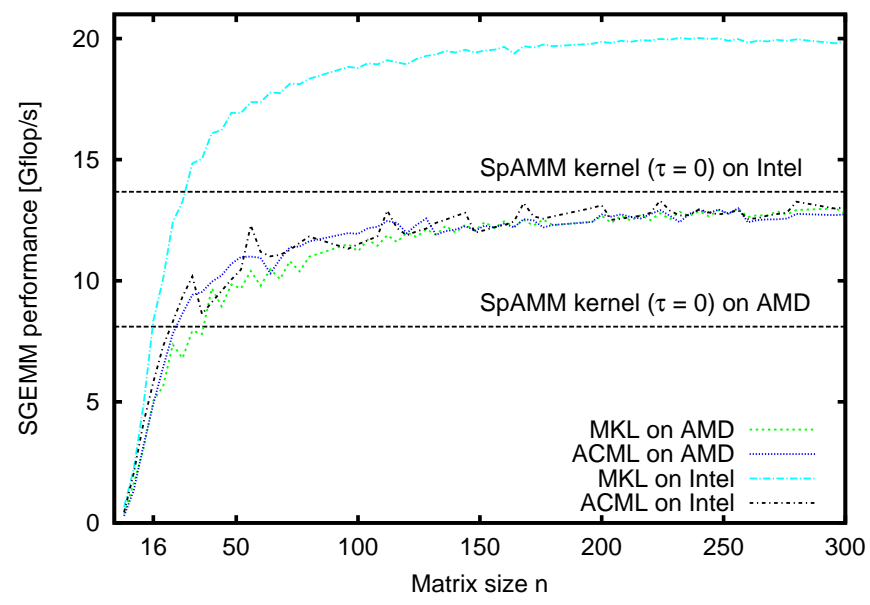

FIG. 3: The performance of SGEMM of the MKL and ACML libraries on AMD Opteron 6168 and Intel Xeon X5650. Clearly shown is the slow ramping up of performance with matrix size. For comparison the average performance on a dense $16 \times 16$ submatrix of our numeric multiply is shown as horizontal lines.

proved data locality, and is typically referred to as cacheoblivious methods [7, 12, 57, 58, 71, 82, 91, 105, 108, 117, 133, 148. Typically, a heuristic such as a SFC is applied to storage and computation ordering as shown in Fig. 1 (C) and (D), which yields excellent locality for both, and the newly gained locality obviates cache-aware methods employed by standard dense methods.

Cache-oblivious algorithms effectively mitigate the issue of latency between memory hierarchies, and would seem to be a natural way to deal with sparse irregular problems such as SpAMM. However, fine grained cache oblivious methods neglect to address the hardware prefetcher, which can have significant impact on application performance [54, 119] and are found to underperform cache-aware approaches for dense linear algebra problems 79, 153 .

\section{B. Hardware Prefetch}

The prefetcher predicts the memory access pattern and preloads the anticipated addresses into cache without software intervention. Currently though, the algorithms employed by the prefetcher are geared towards regular contiguous problems such as dense linear algebra and require fixed stride memory access patterns. In addition, prefetching will not cross a $4 \mathrm{KiB}$ page boundary and the stride has to be within a very short threshold value, which depends on the processor implementation, but is typically 256 or 512 bytes. The prefetcher is triggered after two successive cache misses in the last level cache with address distances within the threshold value 87 , Sec. 2.4.4.4].

In an early implementation of SpAMM using cacheoblivious methods, we concluded that the effects of the 
hardware prefetcher are significant. Our current understanding is that fine grained cache-oblivious techniques introduce variable stride access patterns, which fail to activate the hardware prefetch unit (two successive cache misses with constant stride are necessary), leading to performance degradation relative to conventional cacheaware implementations. This understanding is corroborated by the findings of Bader et al [12] who report poor performance of their cache-oblivious algorithm for DGEMM at fine granularities. While it may be possible to improve fine-grained performance through software prefetch statements, to the best of our knowledge this remains a challenging, unrealized goal for irregular algorithms.

In summary, while it is desirable to apply the SpAMM condition, Eq. (6), aggressively, fine-grained irregular memory access precludes the use of hardware prefetch in its current form, favoring a coarser granularity. Our kernel strikes a compromise between these considerations by implementing storage and computation at different granularities. We retain a quadtree structure down to a granularity of $16 \times 16$ and divide each dense block into $4 \times 4$ submatrices, stored in row-major order but multiplied under the SpAMM condition. While the required conditionals in the kernel degrade performance only slightly in the dense case they lead to increasingly effective performance as measured by the inverse of time-to-solution (see Appendix A), and excellent error control with sparsity. In the following, we detail this strategy.

\section{Recursive Implementation}

An unoptimized version of SpAMM is straightforward to write using recursion and a conventional, vendor tuned kernel for the block multiply. For all matrices with decay in our tests (see Sec. IV B) we found the fastest time to solution for this recursive approach and a conventional SGEMM kernel to occur for $16 \times 16$ blocking.

\section{Fast Implementation}

We divide the SpAMM algorithm into two parts, a symbolic part that collects the submatrices to be multiplied and a numeric part that multiplies the submatrices, described separately in the following.

\section{Symbolic Multiply with Linkless Tree}

Tree data structures and recursive implementations on trees can be inefficient in languages such as $\mathrm{C} / \mathrm{C}++$ or Fortran. While the case of tail-recursion can be inlined by many modern compilers, the general case is significantly more challenging and may not always allow for optimization [128, 132]. Hashed (linkless) tree structures offer a fast and efficient alternative. Instead of storing explicit links, the nodes are stored in hash tables by a linearized

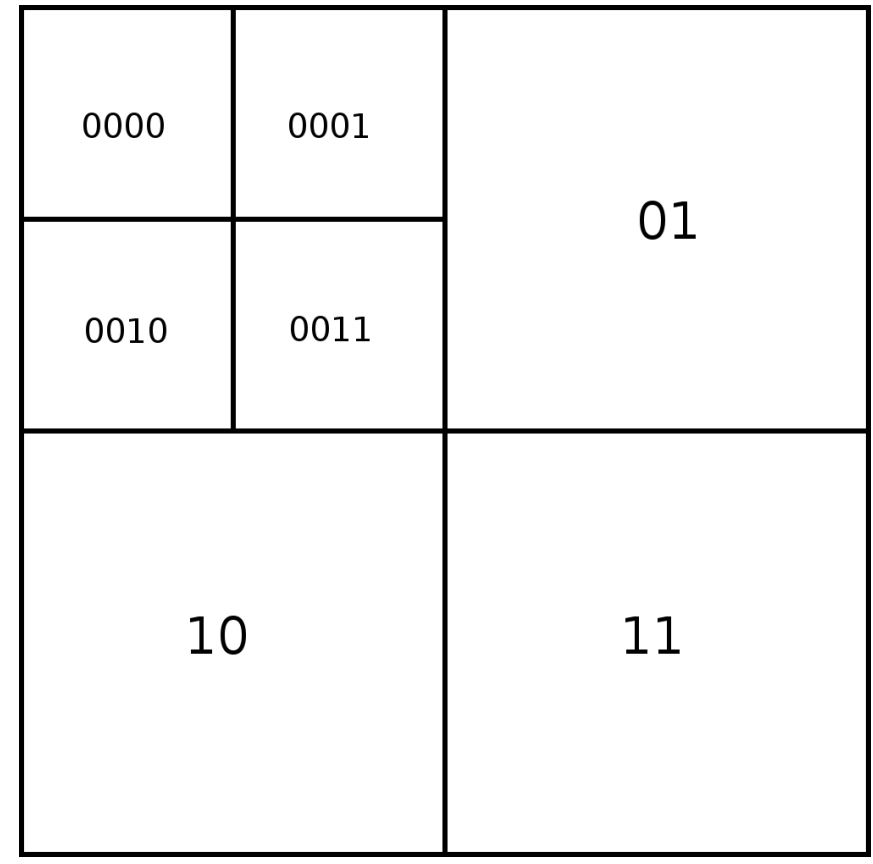

FIG. 4: A schematic representation of the construction of linear quadtree indices and where those indices are in the original matrix. Shown are the linear indices of first tier for the matrix quadrants II, III, and IV. The linear indices of the second tier are shown for quadrant I.

index, as described by Morton 111 and Gargantini 61 and the excellent book by Samet 122 . This technique is often employed in generalized $N$-Body solvers, and in particular in the computer graphics realm for collision detection and culling [10, 24, 47, 96, 97, 99, 155, and in database applications such as the join operation [9, 85, 89, 101, with some of these implemented on GPU systems.

Each tree node is identified by its upper left hand submatrix row- and column indices, $i$ and $j$. In combination with the matrix dimension and the tier depth, the full range of matrix indices covered by a node can be readily reconstructed. The linear index $l$ is calculated through dilating and interleaving $i$ and $j$ :

$$
\begin{aligned}
i & =\sum_{m=0} i_{m} 2^{m} \\
j & =\sum_{m=0} j_{m} 2^{m} \\
l & =\sum_{m=0}\left[i_{m} 2^{2 m+1}+j_{m} 2^{2 m}\right] .
\end{aligned}
$$

For illustration, Fig. 4 shows the first two tiers of linear matrix indices of a small matrix. Notice how each tier adds two digits from the right to the linear index of its parent node and the quadrant labeling scheme is recursively repeated. Despite the lack of links between tree nodes, the full tree structure and its hierarchy can simply be reconstructed on the way down by adding two 
digits from the right, or on the way up by removing those two digits.

It is worth pointing out that for performance the choice of hash function is extremely important. Excessive collision rates are to be avoided, since hash table performance degrades rapidly with increasing collision rate. We use the well designed 32-bit hash function of Jenkins [90, which limits the tree depth to $16+4$ (since we store $16 \times 16$ dense submatrices) and the matrix size to $1048576 \times 1048576$, more than sufficient for the tests presented here. Larger matrices are possible through the use of either a larger hash key or a hybrid approach of linked tree (at the top) and linkless tree (at the bottom 20 tiers).

\section{Symbolic Multiply}

The convolution of the two-dimensional vector spaces into the three-dimensional product space, panels (C) and (D) of Fig. 1, is straightforward to implement through recursively applying Eq. (5), but the situation is more complicated in the case of linkless trees. The hash tables of $A$ and $B$ have to be searched and matching submatrix pairs $A_{i k}$ and $B_{k j}$ have to be found, conditional on Eq. (6), which result in a contribution to the resulting submatrix $C_{i j}$. Related problems are common amongst generalized $N$-Body solvers, such as performing queries on hashed trees to join [9], collide [10], add [142], or in other ways combine elements.

The collection of the submatrices that contribute to the product is carried out in several steps. First, the linear indices $l$ for $A$ and $B$ are extracted from the hash tables of the lowest tier $(16 \times 16$ submatrix level $)$ and stored in an array. From Eq. 10 the indices are given bit-wise by:

$$
l_{A, i k}=\ldots i_{2} k_{2} i_{1} k_{1} i_{0} k_{0}
$$

Second, in a first pass, the index arrays are sorted on their $k$-index values using the merge sort algorithm ${ }^{2}$, resulting in an ordered array partitioned into blocks with identical $k$-values; " $k$-value blocks". Application of the bit-mask Ob...010101 to $l_{A, i k}$ and $0 \mathrm{~b} \ldots 101010$ to $l_{B, k j}$ renders this step straightforward. In a second pass, within each

\footnotetext{
2 Although quicksort is often found to be faster than merge sort in practice, it turns out to be a poor choice in our case: Sorting $n$ elements, merge sort exhibits average and worst-case cost of $\mathcal{O}(n \log n)$, while in comparison quicksort exhibits the same average cost, but slows down to $\mathcal{O}\left(n^{2}\right)$ in the worst-case. For the matrices we used in our tests, quicksort tended to deviate from logarithmic towards quadratic behavior, and merge sort consistently performed better. We speculate that a different choice of hash function could improve the performance of quicksort and the sorting steps in the symbolic part, but the design of a good hash function which exhibits few collisions and preserves logarithmic quicksort behavior is beyond the scope of this work.
}

$k$-value block, the indices are sorted by their associated node norms (the Frobenius norm stored at each node, see Sec. IIB for details) in descending order. Alternatively both sort passes can be combined into one pass by rewriting the sort comparison function to include both $k$-index and norm, however we have not found any performance benefit from this approach.

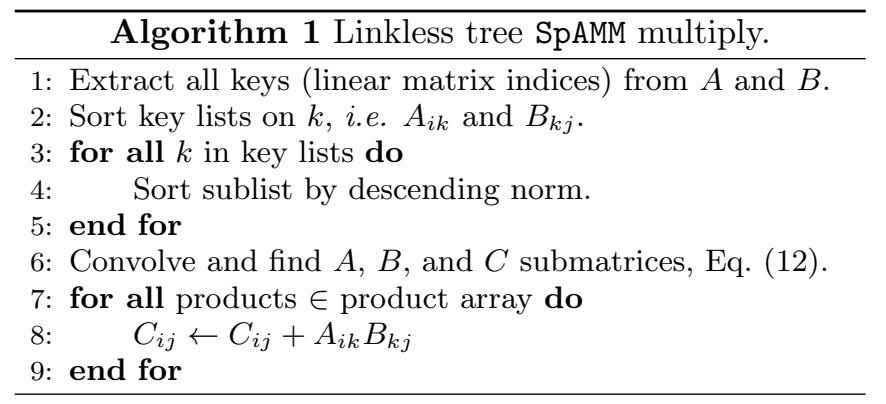

Third, the convolution is performed by nested loops over the $k$-value blocks of the arrays. Although our algorithm only considers the lowest tier, rendering it nonhierarchical, norm sorting the $k$-value blocks restores $\mathcal{O}(n \log n)$ or $\mathcal{O}\left(n^{2} \log n^{2}\right)$ complexity for sparse and dense matrices, respectively. We implemented two versions: The first consists of straightforward nested loops ("nested convolution"), while the second is partially unrolled and uses Intel's SSE intrinsics ("unrolled convolution") that leads to a speed-up of close to three. Since SSE instructions require proper alignment of memory, we align each $k$-value block on a 16-byte boundary and the index computations, the norm products, and the product comparisons are done in SSE, which reduces the loops to a stride of four. We note that the index extraction from the hash tables leads to $\mathcal{O}\left(n^{2}\right)$ storage for the indices of dense matrices. While this is not prohibitive for the matrices tested here, it may become so for larger matrices if not ultimately sparsified. Pointers to the nodes of each triple of submatrices $A_{i k}, B_{k j}$, and $C_{i j}$ are then extracted from the hash tables and appended to an array for further processing by our numeric multiply, described in the following section. The $C_{i j}$ submatrix indices are constructed by masking out the $i$ and $j$ index values of $l_{A, i k}$ and $l_{B, k j}$ and combining the two masked indices,

$$
l_{C, i j}=\left(l_{A, i k} \& 0 \mathrm{~b} \ldots 101010\right) \mid\left(l_{B, k j} \& 0 \mathrm{~b} \ldots 010101\right) .
$$

Steps 1-6 of Algorithm 1 show pseudocode of the convolution step.

While storing the nodes of the linkless tree in a hashtable is very efficient for sparse matrices, a simple array is faster for dense matrices. Since the matrices used in this work are all dense (albeit with the aforementioned decay properties), it is educational to consider a performance comparison between hashtable and array storage of the indices. We therefore benchmarked two implementations of the linkless tree: Hashtable storage of matrix nodes with the nested convolution implementation (labeled "hashtable" in Fig. 5) and array storage of matrix 
nodes with the unrolled convolution implementation using SSE intrinsics (labeled "SSE" in Fig. 5).

\section{Numeric Multiply}

Steps 7-9 of Algorithm 1 show pseudocode for the numeric multiply, and how it serially processes submatrix products. Several factors need to be considered for optimal performance of the kernel: On the one hand, Streaming SIMD Extension (SSE) instructions exhibit higher performance than comparable x87 instructions [87. Sec. 3.8.4]) and in single-precision the SSE vector length is 4 , suggesting a granularity of $4 \times 4$ for applying Eq. 6. On the other hand, this $4 \times 4$ level of granularity leads to inefficient cache use and memory access which can only be remedied by a larger contiguous data structure. Through experimentation, we found $16 \times 16$ contiguous blocks to give the best overall performance. Finally, optimal data storage and access patterns had to be identified. We considered row-major, Morton order, and hierarchical versions of these and found that simple non-hierarchical row-major storage and access is the most efficient.

A note on the hardware prefetch: The $16 \times 16$ singleprecision submatrices occupy 16 cache lines (or more as in our SSE version described below), which is sufficiently large for the hardware prefetch to accelerate memory access. The conditionals around the $4 \times 4$ block multiplies do not significantly degrade performance by themselves, but when products are skipped due to Eq. (6), memory access becomes irregular and prevents the hardware prefetcher from activating, which degrades the flop rate. At the same time however, the effective performance, the inverse of time-to-solution, increases since more products are dropped, more than offsetting the loss of hardware prefetch.

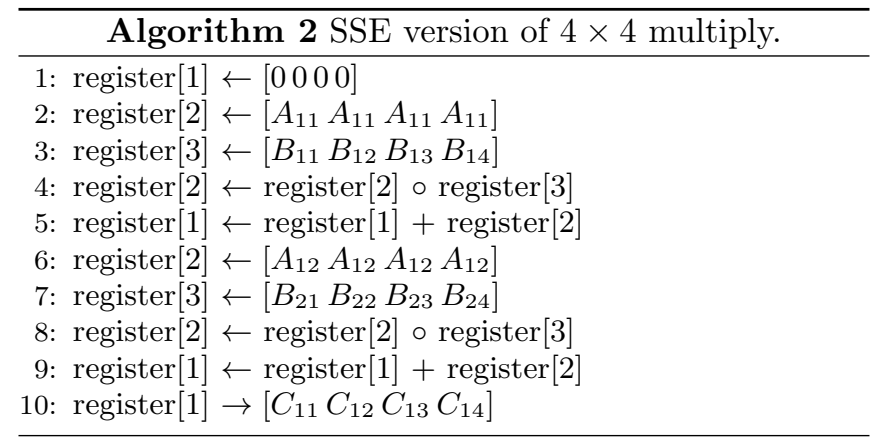

We implemented two versions of the kernel in assembly using the SSE and the SSE4.1 instruction sets. The Xeon X5650 supports both instruction sets, while the Opteron 6168 only has support for SSE. The SSE instruction set was introduced by Intel in 1999 with their Pentium III processor series, adding 70 instructions and eight registers (sixteen on 64-bit processors). Lacking a dot product, our SSE kernel relies on a dilated storage scheme for the matrix elements of $A$, in which each matrix element is stored 4 times, i.e. in vectors such as $\left[A_{11} A_{11} A_{11} A_{11}\right]$. This storage scheme allows for the efficient use of SSE registers without having to shuffle or copy the result matrix elements in SSE, leading to a performance improvement despite the increased demand on memory transfer. We show pseudocode of this kernel version in Algorithm 2. Note that the SSE multiplication and addition instructions used here operate element-wise with the Hadamard or Schur product denoted by the symbol $\circ$.

The SSE4 instruction set was introduced by Intel with their Penryn processor core in 2006. A subset of SSE4, SSE4.1, introduces dot product instructions for packed single-precision numbers and the dilated storage scheme of the SSE version becomes unnecessary. The matrix elements of $B$ are stored in column-major order. Compared to the SSE version, SSE4.1 allows for a reduction of data movement and it uses a dedicated dot product instruction. However, we did not find this version to be faster than the SSE version on the Intel Xeon system.

\section{EXPERIMENTAL METHODOLOGY}

In the following, we give a detailed description of our experimental methodology. In Sec. IV A we will describe in detail the hardware platforms used, and in Sec. IVB the benchmarks used to assess the performance of our implementation.

\section{A. Platforms}

All performance measurements were conducted on two hardware platforms, Intel Xeon X5650 (Westmere microarchitecture, $2.66 \mathrm{GHz}, 6$ cores, $32 \mathrm{KiB}$ of L1 data per core, $256 \mathrm{KiB}$ of L2 per core, $12 \mathrm{MiB}$ L3 shared by all cores) and AMD Opteron 6168 (Magny-Cours microarchitecture, $1.8 \mathrm{GHz}, 12$ cores, $64 \mathrm{KiB}$ L1 data per core, $512 \mathrm{KiB}$ L2 per core, $12 \mathrm{MiB}$ L3 shared by all cores), using a NUMA aware Linux kernel (CONFIG_NUMA and CONFIG_X86_64_ACPI_NUMA) version 2.6.39 on Gentoo 64. Cycle and flop counts where taken with the PAPI library [13, 112, 120] version $4.1 .4^{3}$. Throughout, gcc 63] version 4.4 .5 was used for compilation. It should be noted that the choice of compiler has very little impact on the results of this study because all time intensive computational kernels either are linked in from externally compiled libraries (MKL and ACML) or are written in assembly. Care has to be taken for getting accurate and repro-




ducible measurement results [144; the benchmark process was locked to a processor core and memory allocation was limited to the local NUMA node using the numactl command in combination with the --physcpubind and --membind command line options. In addition all allocated memory was pinned to prevent paging using the mlockall() system call.

\section{B. Benchmarks}

In a typical quantum chemistry calculation, the solution of an integro-differential equation is sought by expanding the electronic wavefunctions of a molecule such as the one shown in panel (A) of Fig. 1 into a set of atomic basis functions yielding a matrix eigenvalue problem [131], or spectral projection [17, 19, 34, 35, 107, 118. The exact form and number of functions in the basis set and hence the number of expansion coefficients per atom depend on the atom type and the basis set. Because SFC ordering is applied atom-wise, matrix elements are clustered by magnitude, grouped in submatrices given by the atom type and basis set. We explore the effect of submatrix size with different basis sets, STO-2G and $6-31 \mathrm{G}^{* *}$, for quantum chemical matrices corresponding to Hilbert curve ordered water clusters. These basis sets introduce submatrices of different sizes (STO-2G: H $1 \times 1$, O $5 \times 5$; $6-31 \mathrm{G}^{* *}: \mathrm{H} 5 \times 5$, O $\left.15 \times 15\right)$, representing the native granularities embedded in the matrix structures. The matrices were generated at the Restricted Hartree-Fock (RHF) level of theory with FreeON, a suite of programs for $\mathcal{O}(N)$ quantum chemistry [25] and are fully converged and without sparsification $(\epsilon=0)$, i.e. the matrices are fully dense but exhibit the approximately exponential decay pattern shown in Fig. 2. The sequence of water clusters corresponds to standard temperature and pressure and have been used in a number of previous studies [31, 38, 40, 41, 49, 109, 116, 125.

The test calculations consist of taking the matrix square, a key step in construction of the spectral projector (density matrix purification) [34, 107, 114, 115, 118. The error of the product is measured by the max norm of the difference to the double-precision (DGEMM) product:

$$
\|\Delta C\|_{\max }=\max _{i j}\left\{\left|C_{i j}-C_{i j}^{\mathrm{DGEMM}}\right|\right\}
$$

which places an upper bound on the element-wise error and is a simple check on the accuracy of the multiplication. For the performance comparisons the tolerance was adjusted to match the errors. While the exact dependence of the product error on $\tau$ is currently unknown, it is worth pointing out that due to its application in product space, $\tau$ affects which product contributions are dropped linearly, as opposed to the quadratic dependence on $\epsilon$ for sparsification 37]. Performance is reported using three metrics, the measured flop rate, the effective performance, and the cycle count as reported by PAPI. For reference, the effective performance is proportional
TABLE I: Performance and error benchmarks

\begin{tabular}{ll}
\hline \hline \multicolumn{1}{c}{ Test } & Label \\
\hline $\begin{array}{l}\text { 1. Comparison of convolution imple- } \\
\text { mentations }\end{array}$ & Symbolic Multiply \\
2. Dense multiply with SGEMM from & SGEMM \\
MKL and ACML (Fig. 3 & \\
3. SpAMM numeric multiply at $4 \times 4$ & $\operatorname{SpAMM~}(4 \times 4)$ \\
4. SpAMM numeric multiply at $16 \times 16$, & $\operatorname{SpAMM}(16 \times 16)$ \\
no conditionals & \\
5. SpAMM multiply at $16 \times 16$ level, & $\operatorname{SpAMM(SGEMM)~}$ \\
SGEMM &
\end{tabular}

to the inverse of the execution time, see Appendix $\mathrm{A}$ for details.

Test 1 consists of a comparison between the different convolution implementations, described in Sec. IIID 2 . Test 2 consists of matrix multiplications with vendor tuned SGEMM for varying matrix sizes to asses the performance of our numeric multiply (Sec. IIID 3). The other tests consist of matrix multiplications with SpAMM to assess the performance and product error of SpAMM. All SpAMM tests use the unrolled convolution implementation with SSE intrinsics, as described in Sec. IIID 2, for the symbolic multiply since it proved to be the fastest, and different versions of the numeric multiply. The numeric multiply used in test 3 applies the SpAMM condition at a $4 \times 4$ submatrix level, which we consider our target granularity. The kernel in test 4 uses a version of the numeric multiply in which the SpAMM conditionals are commented out, which effectively means that the SpAMM condition is applied at a $16 \times 16$ submatrix level. Note that the execution flow is identical to test 3 . The numeric multiply used in test 5 is a vendor tuned SGEMM at a $16 \times 16$ submatrix level. A summary of all benchmark tests can be found in Table 1

\section{RESULTS}

Our results are qualitatively identical on the AMD and the Intel platforms and we will present only the AMD results in the following. In addition, we did not find any difference in the error between MKL and ACML and report for the error, Figs. 6 and 7 , only the MKL results.

\section{A. Symbolic Multiply}

Fig. 5 (test 1 of Table I) shows a cycle count comparison of the symbolic part of $\operatorname{SpAMM}\left(4 \times 4, \tau=2 \times 10^{-8}\right)$ between the recursive and the two linkless tree versions (see Sec. III D 2). For a matrix of $n=7500$, the unrolled convolution using arrays is almost 4.5 times faster than the recursive version and about 2.5 times faster than the 


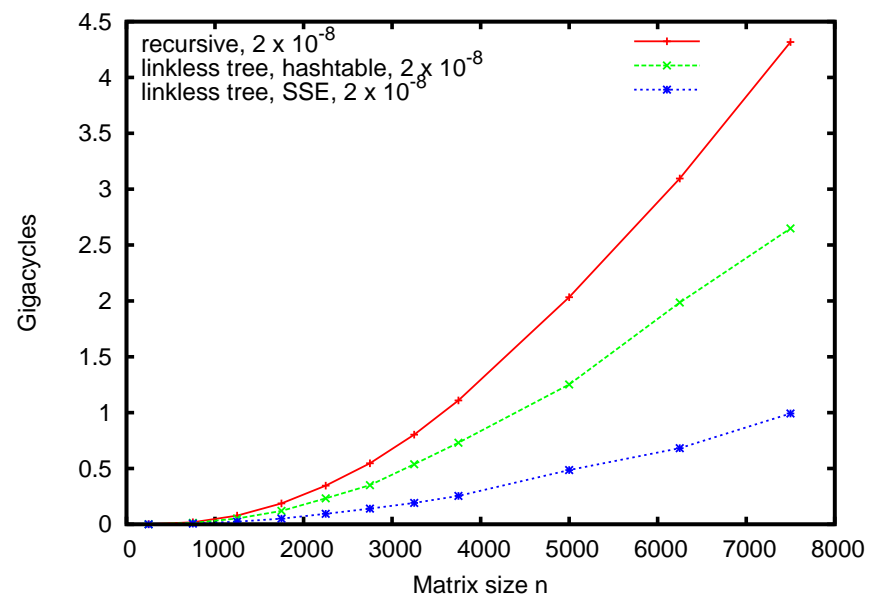

FIG. 5: The cycle counts of the symbolic part of SpAMM with a tolerance of $\tau=2 \times 10^{-8}$ for $\mathrm{RHF} / 6-31 \mathrm{G}^{* *}$ water cluster for the recursive multiply and the two linkless tree implementations.

nested convolution using hashtables. At this matrix size, the symbolic part accounts for about $12 \%$ of the total cost of the multiply.

\section{B. Numeric Multiply vs. SGEMM}

Figure 3 (test 2 of Table I) shows the performance of SGEMM on the Intel and AMD platforms from MKL and ACML. The performance slowly ramps up and asymptotes to $12.6 \mathrm{Gflop} / \mathrm{s}$ for ACML and MKL on AMD and 12.7 Gflop/s for ACML and 19.8 Gflop/s for MKL on Intel. Also shown in the figure the average performance of our numeric multiply on a dense $16 \times 16$ block for large matrices, exhibiting a performance of 8.1 ( $64 \%$ of peak ACML/MKL) and 13.7 (69\% of peak MKL) Gflop/s on AMD and Intel, respectively. At a matrix size of $16 \times 16$, the performance ratio between the SpAMM kernel and SGEMM is $162 \%$ (5.0 Gflop/s for MKL and ACML) for AMD and 161\% (8.5 for MKL) for Intel.

\section{Errors}

In order to facilitate a fair comparison between tests 3,4 , and 5 (Table I), appropriate tolerance values were found to match the matrix errors, Eq. (13), in the different tests. In Figs. 6 and 7 the matrix errors of the test calculations for the two basis sets are shown. The differences in error between tests 4 and 5 were found to be insignificant and results of test 5 are not shown for clarity in these figures. For comparison, a dense multiply exhibits an error of around $10^{-6}$ and we note that $\operatorname{SpAMM}(4 \times 4, \tau=0)$ achieves a smaller error than SGEMM for both basis sets, which is likely due to differences in execution order; hierarchical vs. row-column. For the following tests of SpAMM we chose two error targets, $10^{-6}$

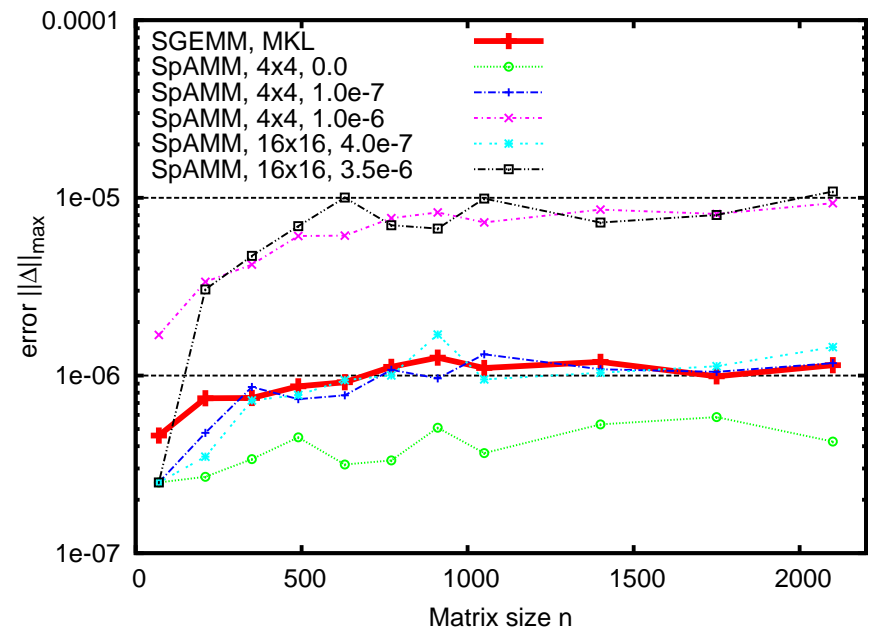

FIG. 6: Error comparison as measured by the max norm, Eq. (13), for water clusters of different sizes in RHF/STO-2G on AMD.

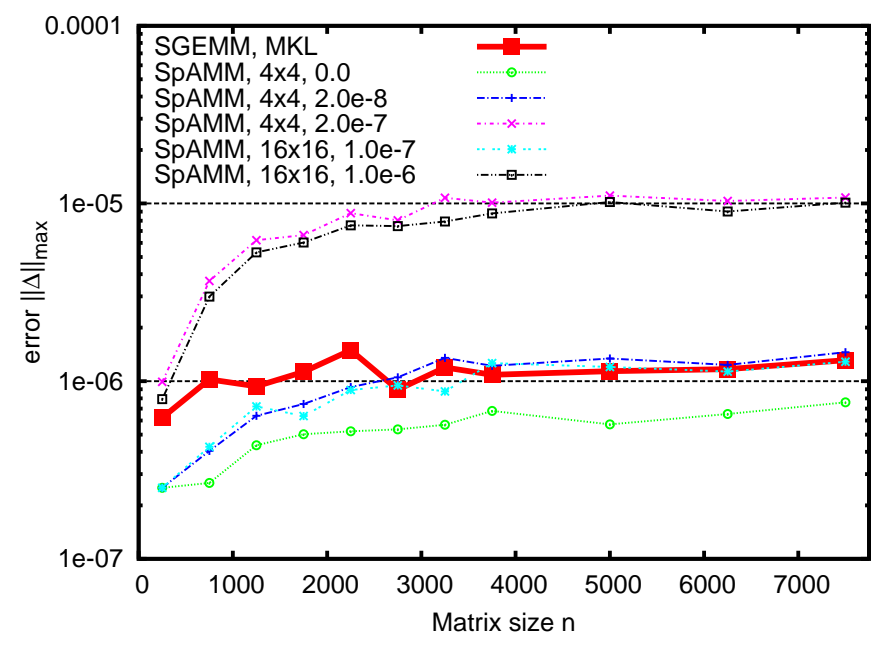

FIG. 7: Error comparison as measured by the max norm, Eq. 13, for water clusters of different sizes in $\mathrm{RHF} / 6-31 \mathrm{G}^{* *}$ on AMD.

and $10^{-5}$, and found the tolerance values shown in Table III Increasing the granularity from $4 \times 4$ to $16 \times 16$ requires an increase of the tolerance by about a factor of 4 for the STO-2G basis set, and a factor of 5 for the 6-31G** basis set.

\section{Effective Performance of SGEMM vs. SPAMM}

Comparing the effective performance of SGEMM, $\operatorname{SpAMM}\left(4 \times 4, \tau=2 \times 10^{-8}\right)$ and $\operatorname{SpAMM}\left(4 \times 4, \tau=2 \times 10^{-7}\right)$, for the RHF $/ 6-31 \mathrm{G}^{* *}$ water clusters in Fig. 8, we find an approximately quadratic performance increase of SpAMM with respect to matrix size. Fits to a quadratic are shown as solid lines. The SGEMM performance is matrix size independent above $n \approx 1000$ due to memory bandwidth saturation and the $\mathcal{O}\left(n^{3}\right)$ complexity of the standard 
TABLE II: The tolerance values identified for the water clusters at different SpAMM granularity and errors.

\begin{tabular}{|c|c|c|c|}
\hline basis set & error & granularity & tolerance \\
\hline \multirow{4}{*}{ STO-2G } & \multirow{2}{*}{$10^{-6}$} & $4 \times 4$ & $1.0 \times 10^{-7}$ \\
\hline & & $16 \times 16$ & $4.0 \times 10^{-7}$ \\
\hline & \multirow{2}{*}{$10^{-5}$} & $4 \times 4$ & $1.0 \times 10^{-6}$ \\
\hline & & $16 \times 16$ & $3.5 \times 10^{-6}$ \\
\hline \multirow{4}{*}{$6-31 \mathrm{G}^{* *}$} & \multirow{2}{*}{$10^{-6}$} & $4 \times 4$ & $2.0 \times 10^{-8}$ \\
\hline & & $16 \times 16$ & $1.0 \times 10^{-7}$ \\
\hline & \multirow{2}{*}{$10^{-5}$} & $4 \times 4$ & $2.0 \times 10^{-7}$ \\
\hline & & $16 \times 16$ & $1.0 \times 10^{-6}$ \\
\hline
\end{tabular}

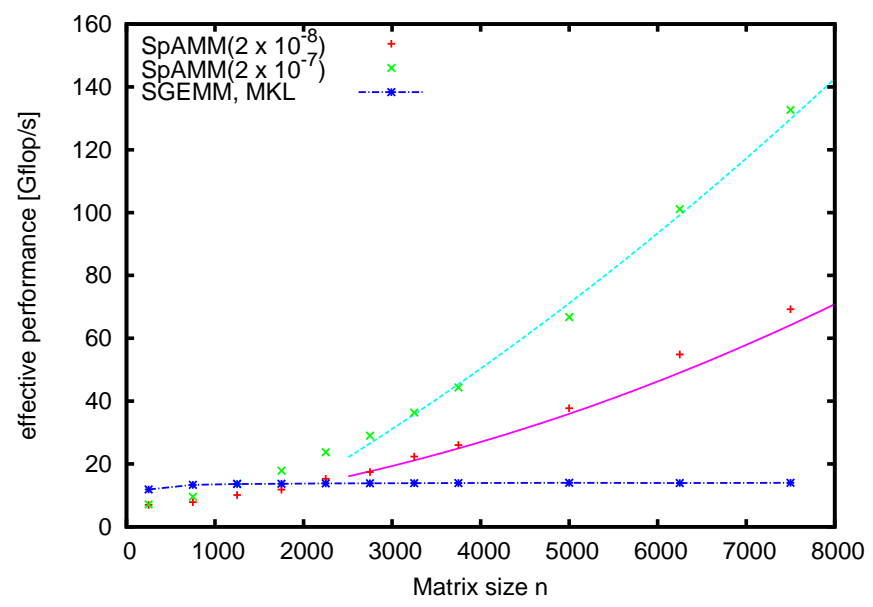

FIG. 8: $\quad$ Effective performance of $\operatorname{SpAMM}\left(4 \times 4, \tau=2 \times 10^{-8}\right)$ and $\operatorname{SpAMM}\left(4 \times 4, \tau=2 \times 10^{-7}\right)$ and SGEMM for the water clusters in $\mathrm{RHF} / 6-31 \mathrm{G}^{* *}$ on AMD. Solid lines show fits to second order polynomial.

matrix multiply. We note the early cross-over of SpAMM at around $n \sim 2000$ and the fact that SpAMM performs at approximately $50 \%$ of peak SGEMM performance as $n \rightarrow 0$ consistent with the results shown in Fig. 3 .

\section{E. Performance of SpAMM}

Figures 9 and 10 show the measured performance of tests 3, 4, and 5 of Table $\mathbb{I}$ for the tolerance values of Table II] Fits to a line are shown to highlight the approximate linear scaling behavior of SpAMM and to give an indication of slope. The cycle count, which is proportional to CPU time, is shown in the main graph, and the effective performance in the inset. For comparison the performance of SGEMM is shown in the inset as a thick line.

For the smaller basis set, $\operatorname{SpAMM}(4 \times 4)$ is about $1.2-$ 1.4 times faster compared to $\operatorname{SpAMM}(16 \times 16)$, with this gain diminishing with increasing tolerance. However, no such performance difference is observed for the larger ba-

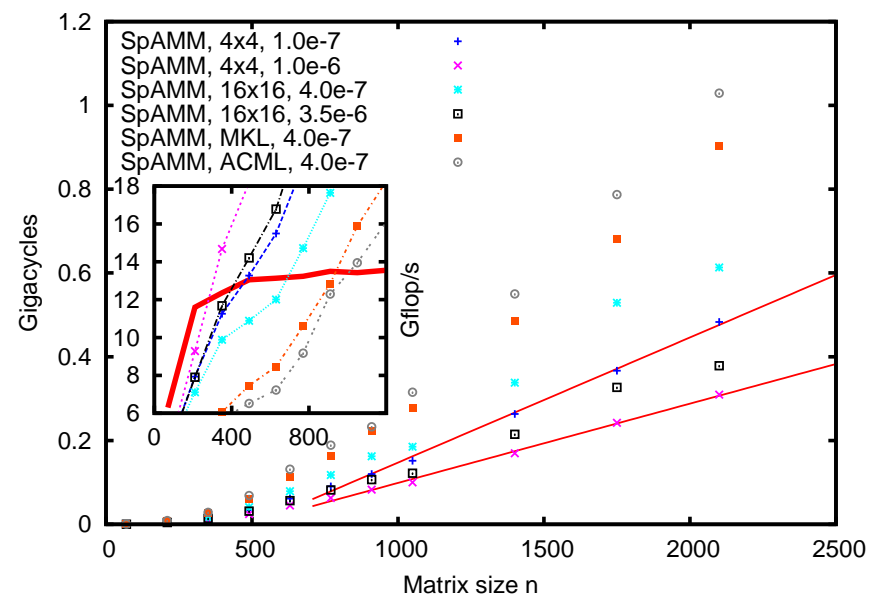

FIG. 9: Performance comparison for water clusters of different sizes in RHF/STO-2G on AMD. Solid lines show fits to a line. Shown in the inset, the effective performance in Gflop/s compared to SGEMM from MKL and ACML (thick line).

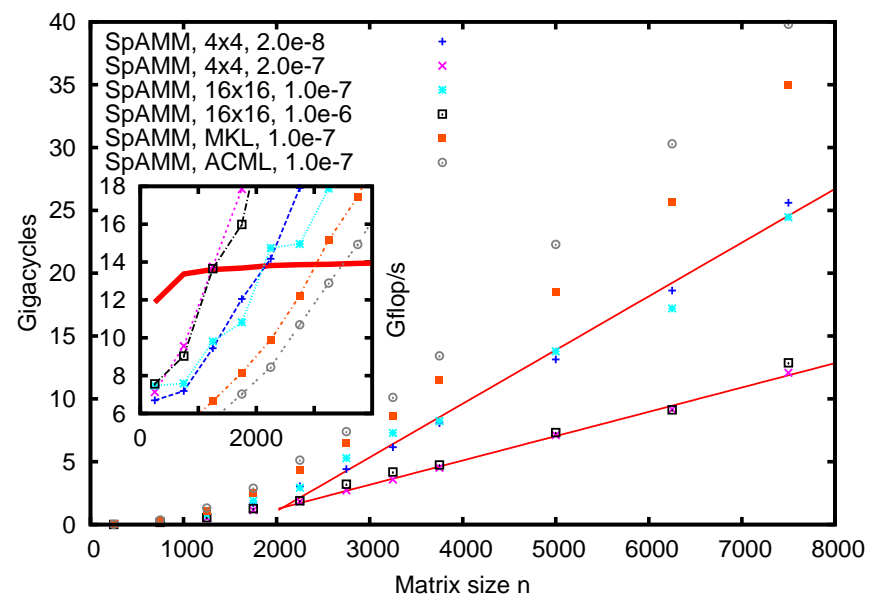

FIG. 10: Performance comparison for water clusters of different sizes in RHF/6-31G** on AMD. Solid lines show fits to a line. Shown in the inset, the effective performance in Gflop/s compared to SGEMM from MKL and ACML (thick line).

sis. $\operatorname{SpAMM(SGEMM)~is~significantly~slower~for~both~error~}$ targets with MKL surprisingly outperforming ACML, the CPU vendor's library. $\operatorname{SpAMM}(4 \times 4)$ and $\operatorname{SpAMM}(16 \times 16)$ achieve approximate linear scaling at $n \approx 600$ in STO$2 \mathrm{G}$ and $n \approx 2000$ in $6-31 \mathrm{G}^{* *}$. For $\mathrm{STO}-2 \mathrm{G}$, crossover between SpAMM and SGEMM occurs at $n \approx 300$ for $\operatorname{SpAMM}(4 \times 4), n \approx 500$ for $\operatorname{SpAMM}(16 \times 16)$, and $n \approx 900$ for $\operatorname{SpAMM}\left(\mathrm{SGEMM}, \tau=4.0 \times 10^{-7}\right)$. For $6-31 \mathrm{G}^{* *}$, crossover occurs at $n \approx 2250$ for $\operatorname{SpAMM}\left(4 \times 4, \tau=2.0 \times 10^{-8}\right)$ and $\operatorname{SpAMM}\left(16 \times 16, \tau=10^{-7}\right), n \approx 1250$ for $\operatorname{SpAMM}(4 \times 4$, $\left.\tau=2.0 \times 10^{-7}\right)$ and $\operatorname{SpAMM}\left(16 \times 16, \tau=10^{-6}\right)$, and $n \approx 3500$ for $\operatorname{SpAMM}\left(\operatorname{SGEMM}, \tau=1.0 \times 10^{-7}\right)$.

Figures 11 and 12 show performance ratios of tests 3 and 4 of Table I for a target error of $10^{-6}$. Shown are the ratios of complexity $\left(C_{16} / C_{4}\right)$, measured flops (flop $16 /$ flop $\left._{4}\right)$, and cycle count $\left(T_{16} / T_{4}\right)$, where we define 


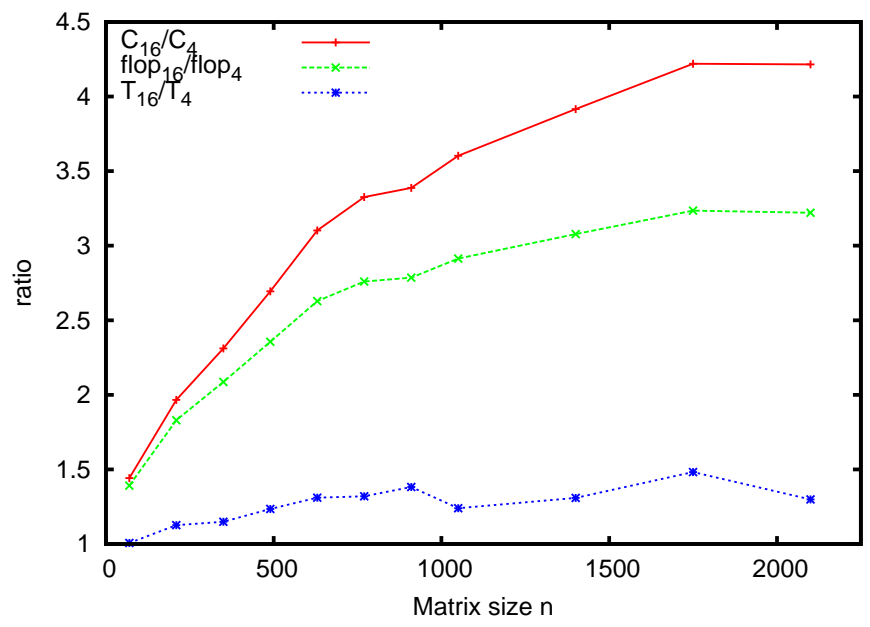

FIG. 11: Performance ratios of $\operatorname{SpAMM}(4 \times 4, \tau=1.0 \times$ $\left.10^{-7}\right)$ and $\operatorname{SpAMM}\left(16 \times 16, \tau=4.0 \times 10^{-7}\right)$ for water clusters of different sizes in RHF/STO-2G on AMD. Shown are the complexity ratio, $C_{16} / C_{4}$ (the number of $4 \times 4 \times 4$ matrix products), the flop ratio, $f_{16} / f_{4}$ (measured), and the cycle count ratio, $T_{16} / T_{4}$.

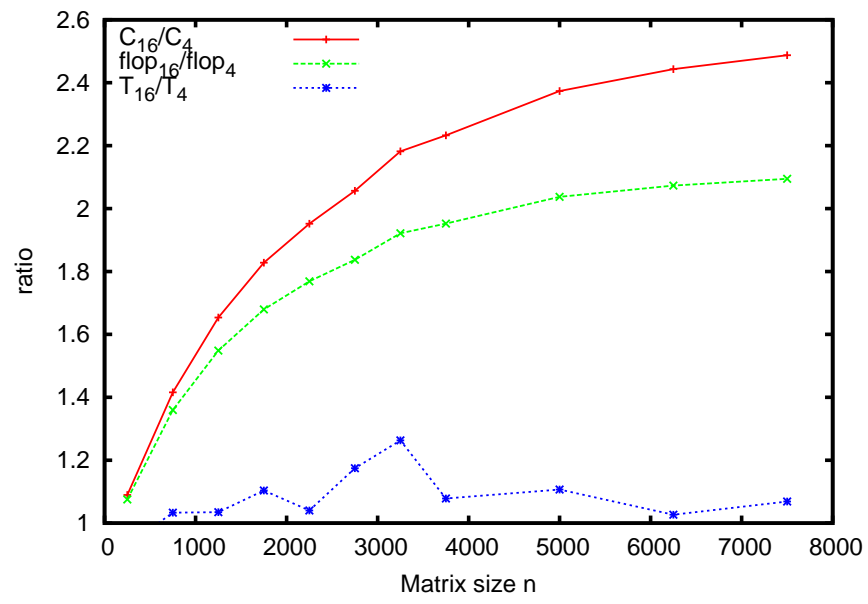

FIG. 12: Performance ratios of $\operatorname{SpAMM}\left(4 \times 4, \tau=2.0 \times 10^{-8}\right)$ and $\operatorname{SpAMM}\left(16 \times 16, \tau=10^{-7}\right)$ for water clusters of different sizes in RHF/6-31G** on AMD.

the complexity as the number of $4 \times 4 \times 4$ matrix multiplications executed, and one $16 \times 16 \times 16$ multiplication translates into a complexity of 64 . For the largest water cluster, the complexity ratio is about 4.5 for STO-2G and 2.5 for $6-31 \mathrm{G}^{* *}$, the flop ratio is about 3.7 for STO- $2 \mathrm{G}$ and 2.1 for $6-31 \mathrm{G}^{* *}$, and the cycle ratio is about 1.3 for STO-2G and 1.1 for $6-31 \mathrm{G}^{* *}$. Note that the difference between complexity and flop ratios is due to the norm product computations in the conditionals.

\section{DISCUSSION}

The error achieved by $\operatorname{SpAMM}\left(4 \times 4, \tau<2.0 \times 10^{-8}\right)$ (Figs. 6 and 7) is lower than SGEMM. We attribute this behavior to superior order of operation associated with hierarchical summation of equi-magnitude blocks. A related problem for future work involves establishing precise relationships between the SpAMM approximation, matrix decay and application specific measures of error. In addition, our implementation can be readily extended to double-precision; however, our goal in this work is to achieve full single-precision (or better) and an early onset of linear scaling.

The storage complexity for dense matrices is $\mathcal{O}\left(n^{2}\right)$ but $\mathcal{O}(n)$ in the sparsified case for matrices exhibiting approximately exponential decay. The computational complexity for convolution is $\mathcal{O}\left(n^{2} \log n^{2}\right)$ in the dense and $\mathcal{O}(n \log n)$ in the sparsified case due to either octree recursion or index sorting in the symbolic multiply, see Fig. 5. The computational complexity of the numeric multiply is $\mathcal{O}(n)$ in either case, when decay is fast enough, or the matrix threshold is large enough. For the matrices studied here, we find that the numeric multiply dominates a small but admittedly quadratic component from the symbolic kernel. Nevertheless we observe an approximately quadratic increase in effective performance, as shown in Fig. 8, with an approximately linear increase in cycle count, as shown in Figs. 9 and 10.

Comparing the cycle counts shown in Figs. 9 and 10 between $\operatorname{SpAMM}(4 \times 4)$ and $\operatorname{SpAMM}(16 \times 16), \operatorname{SpAMM}(4 \times 4)$ holds a slight advantage over $\operatorname{SpAMM}(16 \times 16)$ for both basis sets. We attribute differences to variations between matrix structures; in the STO-2G basis the submatrices of $\mathrm{H}$ and $\mathrm{O}$ are small and $\operatorname{SpAMM}(4 \times 4)$ is able to more fully exploit the matrix structure leading to a bigger performance advantage, while in the $6-31 \mathrm{G}^{* *}$ basis, the submatrices of $\mathrm{H}$ and $\mathrm{O}$ are larger, and $\operatorname{SpAMM}(16 \times 16)$ is more appropriate. We find that the corresponding flop count ratios, flop $16 /$ flop $_{4}$, (shown in Figs. 11 and 12 to be significantly larger than the cycle count ratios, $T_{16} / T_{4}$, clearly indicating the difficulty of translating the reduced complexity of fine grained algorithms into performance gains on commodity platforms. It should be noted that both numeric kernels make full use of SSE, and the issue is not one of inefficient use of floating point resources.

The significant complexity reduction shown in Figs. 11 and 12, as well as the early onset of linear scaling shown in Figs. 9 and 10, underscore the importance of fine granularities. However, the corresponding irregular access prevents the hardware prefetch mechanism in the CPU from activating, resulting in a performance loss due to higher memory latencies, partially offsetting the performance gains due to complexity reduction $(2-3 \mathrm{x}$ in this case). So far we have been unsuccessful in closing this performance gap by using software prefetch statements and $16 \times 16$ blocks, as additional conditionals and the required large distance between prefetch and data lead to no improvement. Indeed, it appears that optimal placement of software prefetch is not only difficult, but that the performance gains are generally limited [11, 46, 86, 150].

Recently much effort has been dedicated to the co- 
design of improved prefetch mechanisms, see for instance the "Scheduled Region Prefetch" 103, 138, "Flux Cache" 62, "Traversal Cache" [127], or the "Block Prefetch Operation" 93. These works support and underscore our conclusion that it is the mechanism of memory access, and not the memory hierarchy per se that is the bottle neck for fine grained irregular algorithms including $\operatorname{SpAMM}(4 \times 4)$, related $N$-body algorithms, as well as the generic pointer chasing problem.

\section{CONCLUSIONS}

We have developed an optimized single-precision implementation of the SpAMM algorithm using linkless trees and SSE intrinsics in combination with a hand-coded assembly kernel, and demonstrated early cross-overs with vendor tuned SGEMMs and an early onset of linear scaling for dense quantum chemical matrices. For small values of the product space threshold $\tau$, we find SpAMM exhibits superior error control relative to SGEMM. While this is unsurprising given the $\mathcal{O}\left(n^{2}\right)$ error scaling for classical matrix-matrix multiplication relative to an $\mathcal{O}(n \log n)$ scaling for recursive multiplication [23, it is worth noting that our fast implementation is doing something else entirely, namely norm sorting prior to convolution (as described in Section IIID 2). The error analysis of this approach, as well as for approximate recursive multiplication of SFC clustered matrices, is an interesting and open problem. Importantly, the issue has been refocused on achieving performance and error control that is superior to the classical multiply, rather than on the errors associated with vector space truncation and their accumulation in classical multiplication.

In this implementation ${ }^{4}$, the matrices are stored in contiguous chunks of size $16 \times 16$, which is unusually small compared to other high performance matrix multiplies. In addition, the norm condition, Eq. (6), is applied to $4 \times 4$ submatrices giving the implementation fine grained error control with a remaining potential for $2-3 \mathrm{x}$ gains in performance on future hardware with improved prefetch. We speculate that these gains might be unlocked with hardware modifications such as the Block Prefetch Operation 93. Nevertheless, relative to naïve implementations of SpAMM using vendor tuned versions of SGEMM, such as those found in Intel's Math Kernel Library (MKL) or AMD's Core Math Library (ACML), our optimized version is found to be significantly faster.

Matrix-matrix multiplication is now aligned with the $N$-Body programming models employed by other quantum chemical solvers [36, 39, 40, and more broadly with the generalized $N$-Body framework [73, 74, 83, 104. As such, its worth noting that SFC ordering in the vector and product space, shown in (C) and (D) of Fig. 1, may also provide a mechanism for domain decomposition and load balance, corresponding to methods that have been already proven for other parallel irregular $N$-Body problems [8, 32, 53, 139, 143]. Likewise, overdecomposition of the recursive, three-dimensional SpAMM task space may provide significant opportunities for parallelism through advanced middleware such as charm++ 92 or tascel 102, 106, as well as through task parallel programming models supported by Cilk Plus [2] or version 3.0 of the OpenMP standard [5]. The low level SIMD optimizations based on linkless trees presented here also may be extended to evolving vector lengths through compiler auto-vectorization and SIMD language extensions such as those provided by the ispc compiler [4, with applicability to AVX, Intel Xeon Phi architectures and GPUs.

\section{Acknowledgements}

This work was supported by the U. S. Department of Energy under Contract No. DE-AC52-06NA25396 and LDRD-ER grant 20110230ER. The friendly and stimulating atmosphere of the Ten Bar Café is gratefully acknowledged. NB gratefully acknowledges the helpful discussions on irc.freenode.net \#asm and linux-assembly@vger. kernel.org. NB and MC gratefully acknowledge helpful discussions with Michele Benzi, Fred Gustavson, and David Wise. Released under LAUR 11-06091.

\section{Appendix A: Effective Performance}

Typically the performance of a solver implementation is measured by the number of CPU cycles (seconds of CPU time), or the number of floating point operations (flop) per cycle (second). While the former directly measures "time to solution", the latter gives insight into the efficiency of the implementation executing computation instructions under the constraints of memory access, register pressure, and other factors. Because of the reduction in flops through Eq. (6), the perceived performance of SpAMM (inverse time to solution) is different than its measured floprate. For comparisons with SGEMM, we therefore model the number of flops by $F=m[k(1+2 n)-n]$ and express the effective performance as the ratio of $F$ and the number of CPU cycles (seconds).
[1] AMD Core Math Library (ACML). http://developer. amd.com/libraries/acml/
[2] Intel Cilk Plus. http://software.intel.com/en-us/ articles/intel-cilk-plus/ 
[3] Intel Math Kernel Library (MKL). http://www.intel. com/software/products/mkl/

[4] Intel SPMD Program Compiler. http://ispc.github. com/

[5] OpenMP. http://openmp.org/

[6] S. Kamal AbDali And David S. Wise, Experiments with quadtree representation of matrices, in Symbolic and Algebraic Computation, P. Gianni, ed., vol. 358 of Lecture Notes in Computer Science, Springer Berlin / Heidelberg, 1989, pp. 96-108.

[7] Michael D. Adams and David S. Wise, Seven at one stroke: results from a cache-oblivious paradigm for scalable matrix algorithms, in Proceedings of the 2006 workshop on Memory system performance and correctness, MSPC '06, New York, NY, USA, 2006, ACM, pp. 41-50.

[8] Srinivas Aluru and Fatih E. Sevilgen, Parallel domain decomposition and load balancing using spacefilling curves, in Proceedings of the 4th IEEE Conference on High Performance Computing, 1997, pp. 230235 .

[9] R. R. Amossen And R. PAGH, Faster join-projects and sparse matrix multiplications, in Proceedings of the 12th International Conference on Database Theory, ACM, 2009, pp. 121-126.

[10] Quentin Avril, Valérie Gouranton, and Bruno ARNALDI, New trends in collision detection performance, in VRIC'09 Proceedings, Simon Richir \& Akihiko Shirai, ed., vol. 11, Laval, France, Apr 2009, p. 53.

[11] A. H. Badawy, A. Aggarwal, D. Yeung, and C. W. Tseng, The efficacy of software prefetching and locality optimizations on future memory systems, Journal of Instruction-Level Parallelism, 6 (2004).

[12] Michael Bader, Robert Franz, Stephan GuenTHER, AND AlEXANDER HEINECKE, Hardware-oriented implementation of cache oblivious matrix operations based on space-filling curves, in Parallel Processing and Applied Mathematics, 7th International Conference, PPAM 2007, Roman Wyrzykowski, Jack Dongarra, Konrad Karczewski, and Jerzy Wasniewski, eds., vol. 4967 of Lecture Notes in Computer Science, Springer, May 2008, p. 628.

[13] E. H. Barragan And J. J. Steves, Performance analisys on multicore system using PAPI, in Computing Congress (CCC), 2011 6th Colombian, may 2011, pp. 1 -5 .

[14] B. J. C. Baxter And George Roussos, A new error estimate of the fast gauss transform, SIAM Journal on Scientific Computing, 24 (2002), pp. 257-259.

[15] P.H. Beckman, Parallel LU decomposition for sparse matrices using quadtrees on a shared-heap multiprocessor, $\mathrm{PhD}$ thesis, Indiana University, 1993.

[16] John Bell, Marsha J. Berger, Jeff Saltzman, AND MiKe WELCOME, Three-dimensional adaptive mesh refinement for hyperbolic conservation laws, SIAM Journal on Scientific Computing, 15 (1994), pp. 127138.

[17] Michele Benzi, Paolo Boito, and Nader Razouk, Decay properties of spectral projectors with applications to electronic structure, arXiv:math.NA, 1203.3953 (2012).

[18] Michele Benzi And Gene H. Golub, Bounds for the entries of matrix functions with applications to preconditioning, BIT, 39 (1999), pp. 417-438.

[19] Michele Benzi And Nader Razouk, Decay bounds and $\mathcal{O}(n)$ algorithms for approximating functions of sparse matrices, Electron. T. Numer. Ana., 28 (2007), p. 16.

[20] Michele Benzi And Miroslav Tuma, Orderings for factorized sparse approximate inverse preconditioners, SIAM J. Sci. Comput., 21 (2000), pp. 1851-1868.

[21] Marsha J. Berger AND P. Colella, Local adaptive mesh refinement for shock hydrodynamics, Journal of Computational Physics, 82 (1989), pp. $64-84$.

[22] Marsha J. Berger And Joseph Oliger, Adaptive mesh refinement for hyperbolic partial differential equations, Journal of Computational Physics, 53 (1984), pp. $484-512$.

[23] Dario Bini AND Grazia LotTi, Stability of fast algorithms for matrix multiplication, Numerische Mathematik, 36 (1980), pp. 63-72.

[24] J. Bittner, V. Havran, ANd P. Slavik, Hierarchical visibility culling with occlusion trees, in Computer Graphics International, 1998. Proceedings, jun 1998, pp. $207-219$.

[25] Nicolas Bock, Matt Challacombe, Chee Kwan Gan, Graeme Henkelman, Karoly Nemeth, AnDers M. N. Niklasson, Anders Odell, Eric Schwegler, C. J. Tymczak, AND Valery Weber, FreeON: A suite of programs for linear scaling quantum chemistry, 2011. http://www.freeon.org/

[26] D. R. BOwler AND T. MiYAzAKI, $O(N)$ methods in electronic structure calculations, arXiv:cond-mat.mtrlsci, 1108.5976 (2011).

[27] D. R. Bowler, T. Miyazaki, and M. J. Gillan, Recent progress in linear scaling ab initio electronic structure techniques, Journal of Physics: Condensed Matter, 14 (2002), p. 2781.

[28] Aydin Buluç And John R. Gilbert, Challenges and advances in parallel sparse matrix-matrix multiplication, in ICPP '08: Proceedings of the 2008 37th International Conference on Parallel Processing, Washington, DC, USA, 2008, IEEE Computer Society, pp. 503-510.

[29] - On the representation and multiplication of hypersparse matrices, in 2008 IEEE International Symposium on Parallel and Distributed Processing, IEEE, Apr. 2008, pp. 1-11.

[30] —, Parallel sparse matrix-matrix multiplication and indexing: Implementation and experiments, Arxiv preprint arXiv:1109.3739, (2011).

[31] John C. Burant, Gustavo E. Scuseria, and MichAEL J. FRISCH, A linear scaling method for hartree-fock exchange calculations of large molecules, The Journal of Chemical Physics, 105 (1996), pp. 89698972.

[32] P. M. Campbell, K. D. Devine, J. E. Flaherty, L. G. Gervasio, And J. D. Teresco, Dynamic octree load balancing using space-filling curves, tech. report, Williams College Department of Computer Science, 2003.

[33] L. E. CAnnon, A Cellular Computer to Implement the Kaiman Filter Algorithm, $\mathrm{PhD}$ thesis, Ph. D. Dissertation, Montana State University, 1969.

[34] Matt Challacombe, A simplified density matrix minimization for linear scaling self-consistent field theory, J. Chem. Phys., 110 (1999), pp. 2332-2342.

[35] — A general parallel sparse-blocked matrix multiply for linear scalingSCF theory., Comp. Phys. Comm., 128 (2000), p. 93. 
[36] Linear scaling computation of the Fock matrix. $V$. hierarchical cubature for numerical integration of the exchange-correlation matrix, J. Chem. Phys., 113 (2000), p. 10037.

[37] Matt Challacombe and Nicolas Bock, Fast multiplication of matrices with decay, arXiv:cs.DS, 1011.3534 (2010).

[38] Matt Challacombe AND ERIC Schwegler, Linear scaling computation of the Fock matrix, J. Chem. Phys., 106 (1997), p. 5526.

[39] Matt Challacombe, Eric Schwegler, AND Jan Almlöf, Computational Chemistry: Review of Current Trends, World Scientific, Singapore, 1996, pp. 53-107.

[40] - Fast assembly of the Coulomb matrix: A quantum chemical tree code, J. Chem. Phys., 104 (1996), pp. 4685-4698.

[41] — Modern Developments in Hartree-Fock Theory: Fast Methods for Computing the Coulomb Matrix, in Computational Chemistry: Reviews of Current Trends, J. Leszczynski, ed., vol. 1 of Computational Chemistry: Reviews of Current Trends, World Scientific, Singapore, 1996, pp. 53-107.

[42] S. Chandrasekaran, M. Gu, and W. Lyons, A fast adaptive solver for hierarchically semiseparable representations, Calcolo, 42 (2005), pp. 171-185.

[43] S. Chandrasekaran, M. Gu, and T. Pals, $A$ Fast \$ULV\$ Decomposition Solver for Hierarchically Semiseparable Representations, SIAM Journal on Matrix Analysis and Applications, 28 (2006), p. 603.

[44] Shimin Chen, Anastassia Ailamaki, Phillip B. GibBONS, AND TODD C. MOWRY, Improving hash join performance through prefetching, ACM Trans. Database Syst., 32 (2007).

[45] H. Cheng, L. Greengard, and V. Rokhlin, A fast adaptive multipole algorithm in three dimensions, Journal of Computational Physics, 155 (1999), pp. 468 498.

[46] T. M. Chilimbi, M. D. Hill, and J. R. Larus, Making pointer-based data structures cache conscious, Computer, 33 (2000), pp. 67-74.

[47] Myung Geol Choi, Eunjung Ju, Jung-Woo Chang, Jehee Lee, And Young J. Kim, Linkless octree using multi-level perfect hashing, Computer Graphics Forum, 28 (2009), pp. 1773-1780.

[48] Don Coppersmith AND ShMuel Winograd, Matrix multiplication via arithmetic progressions, J. Symb. Comput., 9 (1990), pp. $251-280$.

[49] Andrew D. Daniels, John M. Millam, and GusTAVo E. Scuseria, Semiempirical methods with conjugate gradient density matrix search to replace diagonalization for molecular systems containing thousands of atoms, The Journal of Chemical Physics, 107 (1997), pp. $425-431$.

[50] S. Demko, W. F. Moss, And P. W. Smith, Decay rates for inverses of band matrices, Math. Comp., 43 (1984), pp. 491-499.

[51] James W. Demmel, Ioana Dumitriu, Olga Holtz, AND Robert Kleinberg, Fast matrix multiplication is stable, Numer. Math., 106 (2007), pp. 199-224.

[52] James W. Demmel and Nicholas J. Higham, Stability of block algorithms with fast level-3 blas, ACM T. Math. Software, 18 (1992), pp. 274-291.

[53] Karen D. Devine, Erik G. Boman, Robert T. Heaphy, Bruce A. Hendrickson, James D. Teresco,
Jamal Faik, Joseph E. Flaherty, and Luis G. GerVASIO, New challenges in dynamic load balancing, Applied Numerical Mathematics, 52 (2005), pp. 133 - 152. ADAPT '03: Conference on Adaptive Methods for Partial Differential Equations and Large-Scale Computation.

[54] Kevin Dick, A mathematical model of hardware prefetching, in 39th Symposium on Theory of Computing, 2007, p. 1.

[55] J. L. Finkel, R. A. And Bentley, Quad trees a data structure for retrieval on composite keys, Acta Inform., 4 (1974), pp. 1-9.

[56] JeRemy D. Frens And David S. Wise, Auto-blocking matrix-multiplication or tracking blas3 performance from source code, SIGPLAN Not., 32 (1997), pp. 206216.

[57] Matteo Frigo, Charles E. Leiserson, Harald Prokop, AND SRIDHAR RAMACHANDRAN, Cacheoblivious algorithms, in Proceedings of the 40th Annual Symposium on Foundations of Computer Science, FOCS '99, Washington, DC, USA, 1999, IEEE Computer Society, p. 285.

[58] Steven T. Gabriel and David S. Wise, The opie compiler from row-major source to morton-ordered matrices, in Proceedings of the 3rd workshop on Memory performance issues: in conjunction with the 31st international symposium on computer architecture, WMPI '04, New York, NY, USA, 2004, ACM, pp. 136-144.

[59] G. GALLI, Linear scaling methods for Electronic Structure Calculations and Quantum Molecular-Dynamics Simulations, Current Opinion in Solid State \& Materials Science, 1 (1996), pp. 864-874.

[60] Chee Kwan Gan and Matt Challacombe, Linear scaling computation of the fock matrix. vi. data parallel computation of the exchange-correlation matrix, J. Comp. Phys., 118 (2003), pp. 9128-9135.

[61] Irene Gargantini, An effective way to represent quadtrees, Commun. ACM, 25 (1982), pp. 905-910.

[62] G. Gaydadjiev and S. VAssiliadis, Sad prefetching for mpeg 4 using flux caches, Embedded Computer Systems: Architectures, Modeling, and Simulation, (2006), pp. $248-258$.

[63] GCC, the GNU Compiler Collection. http://gcc.gnu. org/

[64] Gentoo, A special flavor of Linux that can be automatically optimized and customized for just about any application or need. http://www.gentoo.org.

[65] Stefan GoedeckeR, Electronic structure methods exhibiting linear scaling of the computational effort with respect to the size of the system, arXiv:cond-mat, 9806073 (1998).

[66] - Linear scaling electronic structure methods, Rev. Mod. Phys., 71 (1999), pp. 1085-1123.

[67] Stefan Goedecker AND G.E. Scuseria, Linear scaling electronic structure methods in chemistry and physics, Computing in Science Engineering, 5 (2003), pp. $14-21$.

[68] Kazushige Goto and Robert A. van de Geijn, On reducing TLB misses in matrix multiplication, Tech. Report CS-TR-02-55, Department of Computer Sciences, The University of Texas at Austin, 2002.

[69] — Anatomy of high-performance matrix multiplication, ACM Trans. Math. Softw., 34 (2008), pp. 12:1$12: 25$. 
[70] - High-performance implementation of the level-3 blas, ACM Trans. Math. Softw., 35 (2008), pp. 4:1-4:14.

[71] Peter Gottschling, David S. Wise, And MichaEL D. ADAMS, Representation-transparent matrix algorithms with scalable performance, in Proceedings of the 21st annual international conference on Supercomputing, ICS '07, New York, NY, USA, 2007, ACM, pp. 116-125.

[72] Lars Grasedyck and Wolfgang Hackbusch, Construction and arithmetics of $\mathcal{H}$-matrices, Computing, 70 (2003), pp. 295-334.

[73] AG GRAY, Bringing Tractability to Generalized NBody Problems in Statistical and Scientific Computation, (2003).

[74] Alexander G Gray and Andrew W Moore, $N$ Body' Problems in Statistical Learning, in Advances in Neural Information Processing Systems, vol. 4, MIT Press, 2001, pp. 521-527.

[75] Leslie Greengard and Vladimir Rokhlin, A fast algorithm for particle simulations, Journal of Computational Physics, 73 (1987), pp. 325 - 348.

[76] — The rapid evaluation of potential fields in three dimensions, in Vortex Methods, Christopher Anderson and Claude Greengard, eds., vol. 1360 of Lecture Notes in Mathematics, Springer Berlin / Heidelberg, 1988, pp. 121-141. 10.1007/BFb0089775.

[77] —, A new version of the Fast Multipole Method for the Laplace equation in three dimensions, Acta Numerica, (1997), pp. 229-269.

[78] Leslie Greengard And John Strain, The fast gauss transform, SIAM Journal on Scientific and Statistical Computing, 12 (1991), pp. 79-94.

[79] J. Gunnels, F. G. Gustavson, K. Pingali, and K. Yotov, Is cache-oblivious dgemm viable?, Applied Parallel Computing. State of the Art in Scientific Computing, (2007), pp. 919-928.

[80] F. G. Gustavson, L. Karlsson, and B. K丸̊gström, Parallel and cache-efficient in-place matrix storage format conversion, ACM Transactions on Mathematical Software, 37 (2011).

[81] Wolfgang Hackbusch And S Börm, Data-sparse approximation by adaptive $\hat{a}$ 2-matrices, Computing, 69 (2002), pp. 1-35.

[82] Philip J. Hanlon, Dean Chung, Siddhartha Chatterjee, Daniela Genius, Alvin R. Lebeck, And ERIN PARKER, The combinatorics of cache misses during matrix multiplication, J. Comput. Syst. Sci., 63 (2001), pp. 80-126.

[83] L. HeRnquist, Hierarchical n-body methods, Computer Physics Communications, 48 (1988), pp. 107-115.

[84] DAVID HilBerT, Über die stetige Abbildung einer Linie auf ein Flächenstück, Math. Ann., (1891), p. 459.

[85] E. G. Hoel And H. Samet, Data-parallel spatial join algorithms, in Parallel Processing, 1994. ICPP 1994. International Conference on, vol. 3, aug. 1994, pp. 227 -234 .

[86] Y. Huang And Z. Gu, Performance comparison of data prefetching for pointer-chasing applications, in Information Science and Engineering (ICISE), 2009 1st International Conference on, IEEE, 2009, pp. 307-310.

[87] InTEL, Intel 64 and IA-32 Architectures Optimization Reference Manual, Intel, November 2009. Order Number: 248966-020.
[88] ArIEH IsERLEs, How large is the exponential of a banded matrix?, J. New Zealand Math. Soc., 29 (2000), p. 177.

[89] Edwin H. Jacox And HanAn SAmEt, Iterative spatial join, ACM Trans. Database Syst., 28 (2003), pp. 230 256.

[90] Bов Jenkins, 32-bit hashes for hash table lookup, May 2006. http://burtleburtle.net/bob/c/lookup3.c

[91] GuOHUA Jin AND John Mellor-CRUMmeY, Using space-filling curves for computation reordering, in Proceedings of the Los Alamos Computer Science Institute, 2005.

[92] L. V. Kale and Sanjeev Krishnan, Charm ++: Parallel programming with message-driven objects, in Parallel Programming using $\mathrm{C}++$, Gregory V. Wilson and Paul Lu, eds., MIT Press, 1996, pp. 175-213.

[93] M. Karlsson, F. DAhlgren, And P. Stenström, A prefetching technique for irregular accesses to linked data structures, in High-Performance Computer Architecture, 2000. HPCA-6. Proceedings. Sixth International Symposium on, IEEE, 2000, pp. 206-217.

[94] Changkyu Kim, Tim Kaldewey, Victor W. Lee, Eric Sedlar, Anthony D. Nguyen, Nadathur Satish, Jatin ChHugani, Andrea Di Blas, AND PradeeP Dubey, Sort vs. hash revisited: fast join implementation on modern multi-core cpus, Proc. VLDB Endow., 2 (2009), pp. 1378-1389.

[95] T. S. LeE, J. P. Lewis, And W. YANG, Linear-scaling quantum mechanical calculations of biological molecules: The divide-and-conquer approach, Computational Materials Science, 12 (1998), pp. 259-277.

[96] Sylvain Lefebvre And Hugues Hoppe, Perfect spatial hashing, ACM Trans. Graph., 25 (2006), p. 579.

[97] - Perfect spatial hashing, in ACM SIGGRAPH 2006 Papers, SIGGRAPH '06, New York, NY, USA, 2006, ACM, p. 579.

[98] David Levinthal, Performance Analysis Guide for Intel Core i7 Processor and Intel Xeon 5500 processors, Intel, 2009.

[99] Thomas Lewiner, Vinícius Mello, Adelailson Peixoto, Sinésio Pesco, And HÉlio Lopes, Fast generation of pointerless octree duals, Computer Graphics Forum, 29 (2010), p. 1661.

[100] Z. Y. LI, W. HE, AND J. L. YANG, Recent progress in density functional theory and its numerical methods, Progress in Chemistry, 17 (2005), pp. 192-202.

[101] Michael D. Lieberman, Jagan SankaraNARAYANAN, AND HANAN SAMET, A fast similarity join algorithm using graphics processing units, in Proceedings of the 2008 IEEE 24th International Conference on Data Engineering, Washington, DC, USA, 2008, IEEE Computer Society, pp. 1111-1120.

[102] J. Lifflander, S. KRIShnamoorthy, AND L.V. KALE, Work stealing and persistence-based load balancers for iterative overdecomposed applications, in Proceedings of the 21st international symposium on High-Performance Parallel and Distributed Computing, ACM, 2012, pp. 137-148.

[103] W. F. Lin, S. K. Reinhardt, and D. Burger, Reducing dram latencies with an integrated memory hierarchy design, in High-Performance Computer Architecture, 2001. HPCA. The Seventh International Symposium on, IEEE, 2001, pp. 301-312.

[104] P. LiU AND S.N. BhATt, Experiences with parallel nbody simulation, in Proceedings of the sixth annual 
ACM symposium on Parallel algorithms and architectures, ACM, 1994, pp. 122-131.

[105] K. Patrick Lorton And David S. Wise, Analyzing block locality in morton-order and morton-hybrid matrices, in Proceedings of the 2006 workshop on Memory performance: Dealing with Applications, systems and architectures, MEDEA '06, New York, NY, USA, 2006, ACM, pp. 5-12.

[106] W. MA AND S. Krishnamoorthy, Data-driven fault tolerance for work stealing computations, in Proceedings of the 26th ACM international conference on Supercomputing, ACM, 2012, pp. 79-90.

[107] R. MCWEENy, The density matrix in self-consistent field theory. $i$. iterative construction of the density matrix, P. Roy. Soc. Lond A Mat., 235 (1956), pp. 496-509.

[108] John Mellor-Crummey, David Whalley, and Ken KENNEDY, Improving memory hierarchy performance for irregular applications using data and computation reorderings, International Journal of Parallel Programming, 29 (2001), pp. 217-247.

[109] John M. Millam and Gustavo E. Scuseria, Linear scaling conjugate gradient density matrix search as an alternative to diagonalization for first principles electronic structure calculations, The Journal of Chemical Physics, 106 (1997), pp. 5569-5577.

[110] Priti Mishra And Margaret H. Eich, Join processing in relational databases, ACM Comput. Surv., 24 (1992), pp. 63-113.

[111] G. M. Morton, A computer oriented geodetic data base and a new technique in file sequencing, tech. report, IBM, Ottawa, 1966.

[112] Philip J. Mucci, Shirley Browne, Christine Deane, and George Ho, Papi: A portable interface to hardware performance counters, in In Proceedings of the Department of Defense HPCMP Users Group Conference, 1999, pp. 7-10.

[113] Aitchiro Nakano, Rajiv K. Kalia, Ken-ichi Nomura, Ashish Sharma, Priya Vashishta, FuyUki Shimojo, Adri C. T. van Duin, William A. GodDARD, RupaK Biswas, AND DeEpaK SRIVAstava, A divide-and-conquer/cellular-decomposition framework for million-to-billion atom simulations of chemical reactions, Computational Materials Science, 38 (2007), pp. $642-652$.

[114] Anders M. N. Niklasson and Matt Challacombe, Density matrix perturbation theory, Phys. Rev. Lett., 92 (2004), p. 193001.

[115] Anders M. N. Niklasson, C. J. TymCZAK, And Matt Challacombe, Trace resetting density matrix purification in $O(N)$ self-consistent-field theory, J. Chem. Phys., 118 (2003), pp. 8611-8620.

[116] Christian Ochsenfeld, Christopher A. White, AND MARTIN HEAD-GORDON, Linear and sublinear scaling formation of hartree-fock-type exchange matrices, The Journal of Chemical Physics, 109 (1998), pp. 1663-1669.

[117] Jesper Holm Olsen and Søren Christian Skov, Cache-oblivious algorithms in practice, master's thesis, Department of Computer Science, University of Copenhagen, Denmark, 2002.

[118] Adam H. R. Palser and David E. ManolopouLOS, Canonical purification of the density matrix in electronic-structure theory, Phys. Rev. B, 58 (1998), pp. 12704-12711.
[119] Shen Pan, Cary Cherng, Kevin DiCK, AND RichARD E. LADNER, Algorithms to take advantage of hardware prefetching, in the Workshop on Algorithm Engineering and Experiments (ALENEX), 2007.

[120] PAPI, Performance Application Programming Interface. http://icl.cs.utk.edu/papi/

[121] G. PEANO, Sur une courbe, qui remplit toute une aire plane, Mathematische Annalen, 36 (1890), pp. 157-160.

[122] Hanan Samet, The design and analysis of spatial data structures, Addison-Wesley Longman Publishing Co., Inc., Boston, MA, USA, 1990.

[123] — Foundations of Multidimensional and Metric Data Structures, Morgan Kaufmann, 2006.

[124] Donovan A. SchneIder AND David J. DeWitt, Tradeoffs in processing complex join queries via hashing in multiprocessor database machines, in Proceedings of the sixteenth international conference on Very large databases, San Francisco, CA, USA, 1990, Morgan Kaufmann Publishers Inc., pp. 469-480.

[125] ERIC Schwegler, Matt Challacombe, and MarTIN HEAD-GORDON, Linear scaling computation of the Fock matrix. II. Rigorous bounds on exchange integrals and incremental fock build, J. Chem. Phys., 106 (1997), pp. 9708-9717.

[126] — A multipole acceptability criterion for electronic structure theory, The Journal of Chemical Physics, 109 (1998), pp. 8764-8769.

[127] Greg Stitt, G. Chaudhari, and J. Coole, Traversal caches: a first step towards fpga acceleration of pointer-based data structures, in Proceedings of the 6th IEEE/ACM/IFIP international conference on Hardware/Software codesign and system synthesis, ACM, 2008, pp. 61-66.

[128] Greg Stitt and Jason Villarreal, Recursion flattening, in Proceedings of the 18th ACM Great Lakes symposium on VLSI, GLSVLSI '08, New York, NY, USA, 2008, ACM, pp. 131-134.

[129] John STRAIn, The fast gauss transform with variable scales, SIAM Journal on Scientific and Statistical Computing, 12 (1991), pp. 1131-1139.

[130] Volker Strassen, Gaussian elimination is not optimal, Numer. Math., 13 (1969), pp. 354-356.

[131] A. Szabo And N.S. Ostuund, Modern quantum chemistry: introduction to advanced electronic structure theory, Dover Pubns, 1996.

[132] PeiYi TAng, Complete inlining of recursive calls: beyond tail-recursion elimination, in Proceedings of the 44th annual Southeast regional conference, ACM-SE 44, New York, NY, USA, 2006, ACM, pp. 579-584.

[133] Jeyarajan Thiyagalingam, Olav Beckmann, and PAUl H. J. KELLY, Improving the performance of morton layout by array alignment and loop unrolling: Reducing the price of naivety, in Proceedings of 16th International Workshop on Languages and Compilers for Parallel Computing, volume 2958 of LNCS, SpringerVerlag, 2003, pp. 241-257.

[134] Robert A. van DE Geijn And J. Watts, Summa: Scalable universal matrix multiplication algorithm, Concurrency Practice and Experience, 9 (1997), pp. 255274.

[135] Joost VandeVondele, Urban Borštnik, And JürG HUTTER, Linear scaling self-consistent field calculations with millions of atoms in the condensed phase, J. Chem. Theory Comput., (2012). 
[136] Virginia VAssilevska Williams, Multiplying matrices faster than coppersmith-winograd, in Proceedings of the 44th symposium on Theory of Computing, STOC '12, New York, NY, USA, 2012, ACM, pp. 887-898.

[137] Xiaoliang Wan and George Em Karniadakis, $A$ sharp error estimate for the fast gauss transform, Journal of Computational Physics, 219 (2006), pp. $7-12$.

[138] Z. Wang, D. Burger, K. S. McKinley, S. K. ReinHARDT, AND C. C. WEEMS, Guided region prefetching: a cooperative hardware/software approach, in ACM SIGARCH Computer Architecture News, vol. 31, ACM, 2003, pp. 388-398.

[139] Michael S. Warren And John K. Salmon, Astrophysical $N$-body simulations using hierarchical tree data structures, in Supercomputing '92, Los Alamitos, 1992, IEEE Comp. Soc., pp. 570-576. (1992 Gordon Bell Prize winner).

[140] - Astrophysical n-body simulations using hierarchical tree data structures, SC Conference, (1992), pp. 570576.

[141] — A parallel hashed oct-tree n-body algorithm, in Proceedings of the 1993 ACM/IEEE conference on Supercomputing, Supercomputing '93, New York, NY, USA, 1993, ACM, pp. 12-21.

[142] — A parallel, portable and versatile treecode, SIAM, Philadelphia, 1995, ch. 1.

[143] — A portable parallel particle program, Computer Physics Communications, 87 (1995), p. 266.

[144] V. M. Weaver And S. A. MCKeE, Can hardware performance counters be trusted?, in Workload Characterization, 2008. IISWC 2008. IEEE International Symposium on, sept. 2008, p. 141.

[145] DAVID S. Wise, Representing matrices as quadtrees for parallel processors: extended abstract, SIGSAM Bull., 18 (1984), pp. 24-25.

[146] - Ahnentafel indexing into morton-ordered arrays, or matrix locality for free, in Euro-Par 2000 Parallel
Processing, Arndt Bode, Thomas Ludwig, Wolfgang Karl, and Roland Wismüller, eds., vol. 1900 of Lecture Notes in Computer Science, Springer Berlin / Heidelberg, 2000, pp. 774-783.

[147] DAVId S. WiSe AND John Franco, Costs of quadtree representation of nondense matrices, Journal of Parallel and Distributed Computing, 9 (1990), pp. $282-296$.

[148] David S. Wise, Jeremy D. Frens, Yuhong Gu, AND GREgORY A. AlexANDER, Language support for morton-order matrices, SIGPLAN Not., 36 (2001), pp. 24-33.

[149] C. Yang, R. Duraiswami, and N Gumerov, Improved Fast Gauss Transform, Tech. Report CS-TR4495, Dept. of Computer Science, University of Maryland, College Park, 2003.

[150] C. L. Yang And A. R. LeBeck, Push vs. pull: Data movement for linked data structures, in Proceedings of the 14th international conference on Supercomputing, ACM, 2000, pp. 176-186.

[151] W. YANG, Direct calculation of electron density in density-functional theory, Phys. Rev. Lett, 66 (1991), pp. 1438-1441.

[152] W. YAng And T. S. LeE, A density-matrix divide-andconquer approach for electronic structure calculations of large molecules, J. Chem. Phys., 103 (1995), p. 5674.

[153] K. Yotov, T. Roeder, K. Pingali, J. Gunnels, And F. Gustavson, An experimental comparison of cacheoblivious and cache-conscious programs, in Proceedings of the nineteenth annual ACM symposium on Parallel algorithms and architectures, ACM, 2007, pp. 93-104.

[154] Raphael Yuster AND URI Zwick, Fast sparse matrix multiplication, ACM T. Alg., 1 (2005), pp. 2-13.

[155] Yi-Sheng Zou, Guo-Fu Ding, Ming-Heng Xu, And Yong HE, Survey on real-time collision detection algorithms, Application Research of Computers, 25 (2008), p. 8 . 